\title{
HP1a is a chromatin crosslinker that controls nuclear and mitotic chromosome mechanics
}

Amy R. Strom ${ }^{\star 1}$, Ronald J. Biggs ${ }^{* 2}$, Edward J. Banigan ${ }^{\star 3}$, Xiaotao Wang $^{4,5}$, Katherine Chiu ${ }^{8}$, Cameron Herman ${ }^{2}$, Jimena Collado ${ }^{2}$, Feng Yue ${ }^{4,5}$, Joan C. Ritland Politz ${ }^{6}$, Leah J. Tait ${ }^{6}$, David Scalzo ${ }^{6}$, Agnes Telling $^{6}$, Mark Groudine ${ }^{6}$, Clifford P. Brangwynne ${ }^{1}$, John F. Marko ${ }^{2,7}$, Andrew D. Stephens ${ }^{8, \#}$

*These authors contributed equally to this work

\#Correspondence: Andrew Stephens (andrew.stephens@umass.edu)

${ }^{1}$ Howard Hughes Medical Institute, Department of Chemical and Biological Engineering, Princeton University, Princeton, NJ 08540, USA.

${ }^{2}$ Department of Molecular Biosciences, Northwestern University, Evanston, IL 60208, USA

${ }^{3}$ Institute for Medical Engineering and Science and Department of Physics, Massachusetts Institute of Technology, Cambridge, MA 02139, USA

${ }^{4}$ Department of Biochemistry and Molecular Genetics, Feinberg School of Medicine, Northwestern University, Chicago, IL 60611, USA.

${ }^{5}$ Robert H. Lurie Comprehensive Cancer Center, Feinberg School of Medicine, Northwestern University, 303 East Superior Street, Chicago, IL 60611, USA

${ }^{6}$ The Fred Hutchinson Cancer Research Center, Seattle, WA 98019, USA

${ }^{7}$ Department of Physics and Astronomy, Northwestern University, Evanston, IL 60208, USA

${ }^{8}$ Biology Department, University of Massachusetts Amherst, Amherst, MA 01003, USA 


\section{Abstract}

Chromatin, which consists of DNA and associated proteins, contains genetic information and is a mechanical component of the nucleus. Heterochromatic histone methylation controls nucleus and chromosome stiffness, but the contribution of heterochromatin protein HP1a (CBX5) is unknown. We used a novel HP1a auxin-inducible degron human cell line to rapidly degrade HP1a. Degradation did not alter transcription, local chromatin compaction, or histone methylation, but did decrease chromatin stiffness. Single-nucleus micromanipulation reveals that HP1 $\alpha$ is essential to chromatin-based mechanics and maintains nuclear morphology, separate from histone methylation. Further experiments with dimerization-deficient HP1 $\alpha^{165 E}$ indicate that chromatin crosslinking via HP1 $\alpha$ dimerization is critical, while polymer simulations demonstrate the importance of chromatin-chromatin crosslinkers in mechanics. In mitotic chromosomes, HP1 $\alpha$ similarly bolsters stiffness while aiding in mitotic alignment and faithful segregation. HP1a is therefore a critical chromatin-crosslinking protein that provides mechanical strength to chromosomes and the nucleus throughout the cell cycle and supports cellular functions. 


\section{Introduction}

Chromatin, which fills the nucleus, is a repository of information, but is also a physical element that provides structure, mechanical rigidity, shape, and function to the nucleus. Heterochromatin is the stiff, compact, and gene-poor form of chromatin. Heterochromatin loss results in abnormal nuclear morphology, which is a hallmark of human disease (Stephens et al., 2019a; Uhler and Shivashankar, 2018). Increasing the amount of heterochromatin by elevating histone methylation levels can increase nuclear stiffness and restore nuclear shape and function in perturbed model cell lines and patient cells of human diseases (Liu et al., 2018; Stephens et al., 2019b, 2018, 2017). Chromatin stiffness also plays a key role during cell division, as mitotic chromosome mechanics are key to the proper segregation of the genome during mitosis (Batty and Gerlich, 2019; Ribeiro et al., 2009; Stephens et al., 2011; Sun et al., 2018). Recently, it has been reported that methylated histones/heterochromatin are also a mechanical component of mitotic chromosomes (Biggs et al., 2019). However, in addition to methylated histones, protein "readers" of epigenetic marks play a key role in defining heterochromatin (and euchromatin). A key histone

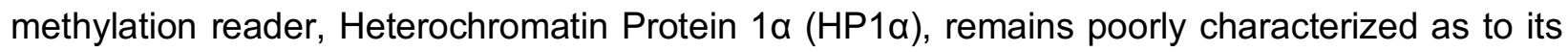
role in controlling the mechanical properties of heterochromatin. To what degree HP1 $\alpha$ contributes to the mechanical resistive capabilities of chromatin, how this contribution is intertwined with histone methylation, and how these result in proper nuclear and mitotic mechanics and function, are all open questions.

HP1 $\alpha$ is a major component of constitutive heterochromatin (James and Elgin, 1986; Singh et al., 1991; Wreggett et al., 1994). Functionally, HP1 $\alpha$ is a homodimer that binds to both DNA and to $\mathrm{H} 3 \mathrm{~K} 9 \mathrm{me}^{2,3}$ constitutive heterochromatin marks. The direct association of HP1 $\alpha$ with $\mathrm{H} 3 \mathrm{~K} 9 \mathrm{me}^{2,3}$ heterochromatin and its direct binding to Suv39h1/2, the histone methyltransferase that deposits $\mathrm{H} 3 \mathrm{~K} 9 \mathrm{me}^{2,3}$, has led to reports that HP1a is necessary for either maintenance or establishment of histone methylation (Bannister et al., 2001; Krouwels et al., 2005).

Loss of HP1a could therefore indirectly alter chromatin mechanics by modulating histone methylation levels. Alternatively, HP1 1 homodimerization and/or higher-order oligomerization could directly impact mechanics through physical bridging of two chromatin fibers, resulting in crosslinking of DNA or H3K9me ${ }^{2,3}$-marked nucleosomes (Canzio et al., 2011; Cheutin et al., 2003; Machida et al., 2018). Consistent with this possibility, the presence of chromatin crosslinks has been shown to be a key element of chromatin organization and mechanics (Banigan et al., 2017; Belaghzal et al., 2019; Lionetti et al., 2020; Stephens et al., 2017). The capacity of HP1 $\alpha$ to drive liquid-liquid phase separation (Larson et al., 2017; Strom et al., 2017) could also contribute to altered chromatin organization and mechanics, given the emerging evidence for links between phase separation and nuclear mechanics (Shin et al., 2018). These mechanisms could also affect mechanics in mitotic chromosomes, where HP1a is also present (Akram et al., 2018; Serrano et al., 2009). The role of HP1 $\alpha$ in controlling chromatin mechanics during both interphase and mitosis, as well as the functions of HP1a-mediated chromatin mechanics, remain to be determined. 
Nuclear and mitotic chromosome micromanipulation force measurements have been critical to understanding the mechanical properties of chromatin, making these techniques ideal for probing the relative roles of histone modifications and chromatin-binding proteins. Nucleus micromanipulation force measurements provide a novel capability, allowing the separation of chromatin, which dominates the initial force response regime, from the other major mechanical component, lamin A, which dictates strain stiffening in the long-extension regime (Stephens et al., 2017). This two-regime force response was recently verified by AFM-SPIM force measurement technique (Hobson et al., 2020). Chromatin-based nuclear mechanics are dictated by euchromatin and heterochromatin levels, particularly through post-translational modifications of histones by acetylation or methylation, respectively (Heo et al., 2016; Hobson et al., 2020; Krause et al., 2019; Liu et al., 2018; Nava et al., 2020; Stephens et al., 2019b, 2018, 2017). These changes in chromatin-based nuclear mechanics can, independently of lamins, cause abnormal nuclear morphology, which is a hallmark of human disease (Stephens et al., 2019a). A recent high throughput screen revealed that many key chromatin proteins also contribute to nuclear shape (Tamashunas et al., 2020), raising the question of the relative roles of histone modifications versus chromatin proteins such as HP1a.

Recent experimental and modeling studies suggest chromatin proteins, like HP1a, may contribute to mechanics by acting as physical linkers. Experimental data for nuclear mechanical response can only be reconciled with models which contain chromatin (an interior polymer), lamina (a peripheral meshwork), and also chromatin-chromatin and chromatin-lamina linkages (Banigan et al., 2017; Hobson and Stephens, 2020; Stephens et al., 2017). Further studies have suggested that these linkages may govern nuclear shape stability (Lionetti et al., 2020; Liu et al., 2020; Schreiner et al., 2015). Experimental studies have shown chromatin linkages to the nuclear periphery aid shape stability and mechanics (Schreiner et al., 2015). Furthermore, recent chromatin conformation capture $(\mathrm{Hi}-\mathrm{C})$ and mechanics experiments suggest that chromatin is physically linked about every $15 \mathrm{~kb}$, since chromatin organization and mechanical response are perturbed only upon extreme chromatin fragmentation by restriction enzymes (Belaghzal et al., 2019). Whether chromatin-binding proteins like HP1a provide mechanical and morphological stability to the nucleus and whether their function is to maintain histone modifications or act as physical linkers, remains an open question.

Most studies of epigenetic modification of chromatin and nuclear mechanics have focused on the interphase nucleus. However, it is conceivable that some of the epigenetic marks involved in heterochromatin formation during interphase might survive and have effects during cell division. Consistent with this, recent work indicates that hypermethylation of histones can persist into metaphase and is correlated with increased stiffness of mitotic chromosomes, i.e., metaphase chromatin (Biggs et al., 2019). However, it remains unknown whether the readers of those marks, i.e., HP1a, contribute significantly to metaphase chromatin structure and mechanics and how important they are to ensuring the success of mitosis.

Here, we determine the mechanical role of well-known constitutive heterochromatin protein HP1 $\alpha$ as it relates to histone methylation. We created and characterized an auxin-inducible-degradation (AID, (Nishimura et al., 2009)) system for acute depletion of endogenous HP1a in human U2OS 
cells. Using these novel CRISPR-derived HP1a-AID-sfGFP cells, we find that the transcriptional profile and chromatin organization are largely unchanged by rapid degradation of HP1a. However, rapid HP1 1 degradation causes decreased chromatin-based rigidity in both nuclei and mitotic chromosomes. Concurrently, we observe increases in aberrant nuclear morphology and incidence of mitotic errors, both of which are associated with disease. Increasing histone methylation rescues nuclear and mitotic chromosome mechanics associated with HP1 $\alpha$ depletion, indicating that these factors contribute independently. Rescue experiments with a HP1 a mutant protein reveal that its dimerization is essential for the maintenance of nuclear structure. Computational modeling supports the conclusion that HP1a's contribution to nuclear mechanics follows primarily from its function as a chromatin-chromatin crosslinker, suggesting that constitutive heterochromatin may be thought of as a polymer gel (Colby et al., 1993). These findings contribute to our understanding of the role of histone methylation and heterochromatin levels and epigenetic marks in controlling nuclear organization, mechanics and morphology, in healthy and diseased cell states. 


\section{Results}

\section{Rapid degradation of HP1a using an auxin-inducible degron}

We generated a novel endogenous HP1a auxin-inducible degron for rapid and reversible depletion of HP1a protein in the cell. This was accomplished using CRISPR (Doudna and Charpentier, 2014) to tag both endogenous copies of the CBX5 gene in U2OS cells with an auxininducible degron (AID, (Nishimura et al., 2009)) and reporter Superfolder Green Fluorescent Protein (sfGFP) at the $\mathrm{C}$ terminus (HP1a-AID-sfGFP). Immunostaining demonstrated that modification of the endogenous loci did not alter the HP1 $\alpha$ protein localization pattern (Figure 1A), and PCR, Western blotting, and flow cytometry showed that all endogenous CBX5 alleles were tagged and only modified protein was expressed (Figure $1 \mathrm{C}$ and 1D, Materials and Methods). HP1a degradation was observed by fluorescence microscopy or flow cytometry of HP1a-AIDsfGFP cells and by Western blot after four hours after treatment with $1 \mathrm{mM}$ auxin (Indole-3-acetic acid, Figure 1, B-D). These conditions consistently resulted in $>90 \%$ degradation of HP1 a (Figure 1,2 , and 5). The degradation was reversible as protein levels recovered over 2 days after removal of auxin (Figure 1, B-D). Thus, we report the novel generation of an endogenously tagged HP1 $\alpha$ cell line providing a fluorescence reporter and capable of rapid degradation in hours that is also reversible.

Previous studies show that disruption of HP1a binding and localization through RNAi knockdown of its binding partners results in chromatin decompaction and loss of transcriptional silencing (Frescas et al., 2008; Hahn et al., 2013; Shumaker et al., 2006). Because tethering of HP1a to specific sites is sufficient to induce chromatin compaction and transcriptional silencing (Li et al., 2003; Verschure et al., 2005), we sought to determine whether, acute depletion of HP1a by auxin treatment would significantly alter transcription or chromatin organization. RNA-Seq data was acquired, mapped (STAR), quantified (RSEM), and the differential gene expression analysis was performed using DESeq2 for greater than 16,500 genes (see Materials and Methods). Transcription analysis of HP1a-AID-sfGFP control and 4 hour auxin treated cells revealed that only 3 genes were downregulated and only 37 genes were upregulated (q-value $<0.05$ and foldchange $>2$, Figure 1E). Lack of transcriptional changes upon acute degradation of HP1a was further supported by comparing control and 16 hours of auxin treatment, which yielded 15 downregulated and 4 upregulated genes (data not shown). These data suggest that satellite derepression and other transcriptional changes previously reported after redistribution of HP1 $\alpha$ may be indirect or may be dependent on secondary chromatin rearrangements due to slower processes, such as epigenetic changes or cell cycle progression like replication or mitosis. DAPI and Hoechst staining patterns showed similar dense regions of nuclear stain typical of heterochromatin in both treated and untreated cells (Figure 1 and data not shown). Furthermore, histone density and distribution do not significantly change on a single-cell basis (Figure 1 - figure supplement 1). These results indicated that no global change in transcription or dissolution of heterochromatin occurred after acute HP1 $\alpha$ degradation. 

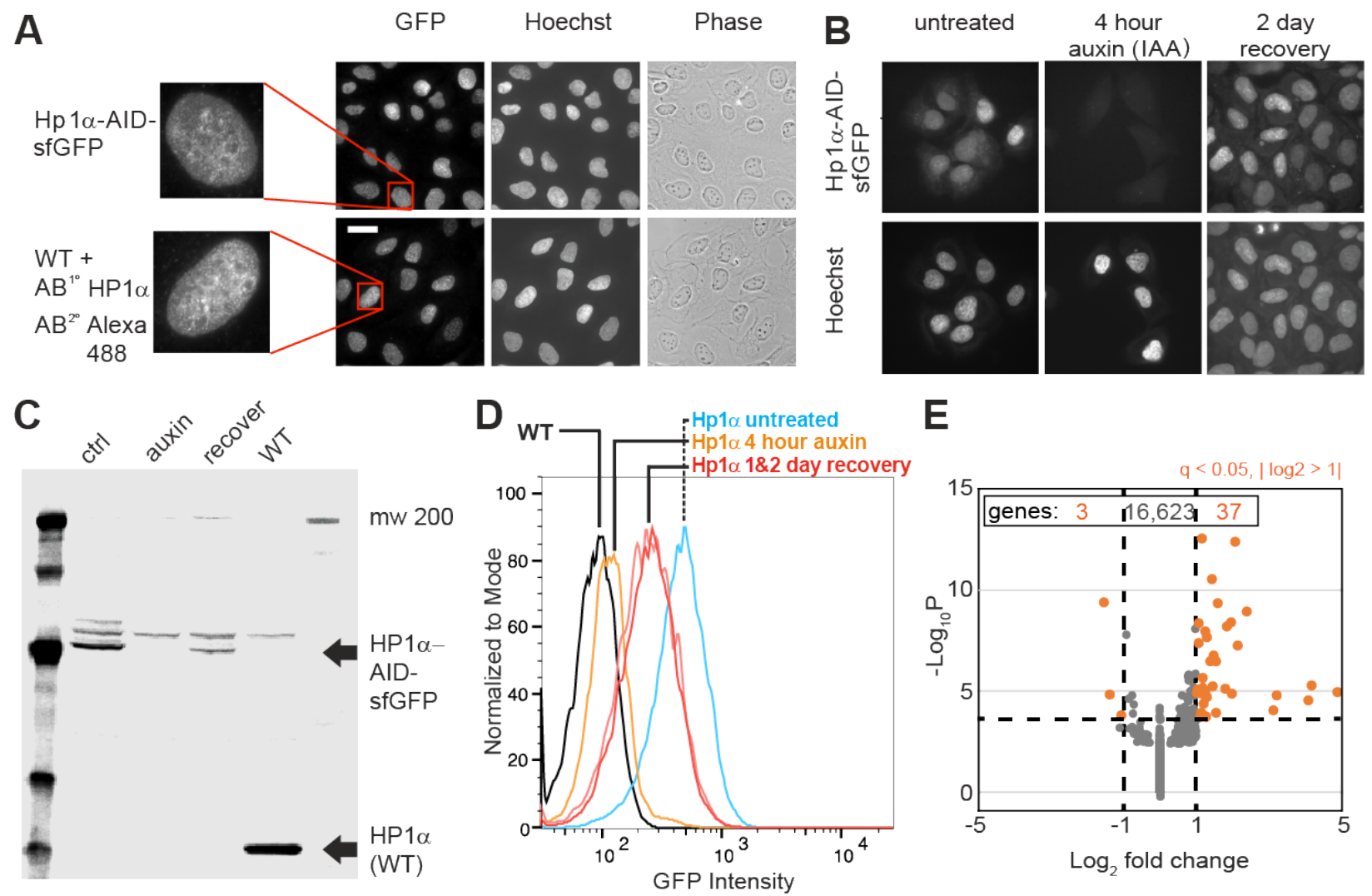

Figure 1. Generation of an CRISPR endogenous HP1 $\alpha$-auxin-inducible-degron-sfGFP cell line.

(A) Example images of HP1a-AID-sfGFP relative to wild-type cells stained for HP1a via immunofluorescence along with Hoechst DNA stain and phase images. Scale bar $=20 \mu \mathrm{m}$. (B) Example images of HP1 $\alpha$-AID-sfGFP before, after 4 hours of auxin treatment, and two days post auxin removal. Hoechst DNA stain aids labeling of nuclei. (C) Western blot and (D) Flow cytometer graph of GFP intensity of control (ctrl/untreated), auxin treated for 4 hours, two days after removal of auxin, and wild-type (WT) showing acute loss of recovery of HP1a-AID-sfGFP. Quantification of HP1a-AID-sfGFP shows $90 \%$ degradation by fluorescence microscopy in Figure $2 \mathrm{~F}$ and Figure 5A. (E) Graph of RNA-seq data showing that few genes change transcript levels as determined by q-value $<0.05$ (calculated via $-\log _{10} P$ ) and absolute change of Log2 fold $>1$ (marked in orange), with expression of only 40 out of of 16,663 genes changing significantly comparing control/untreated versus 4 hour auxin-treated. 
A
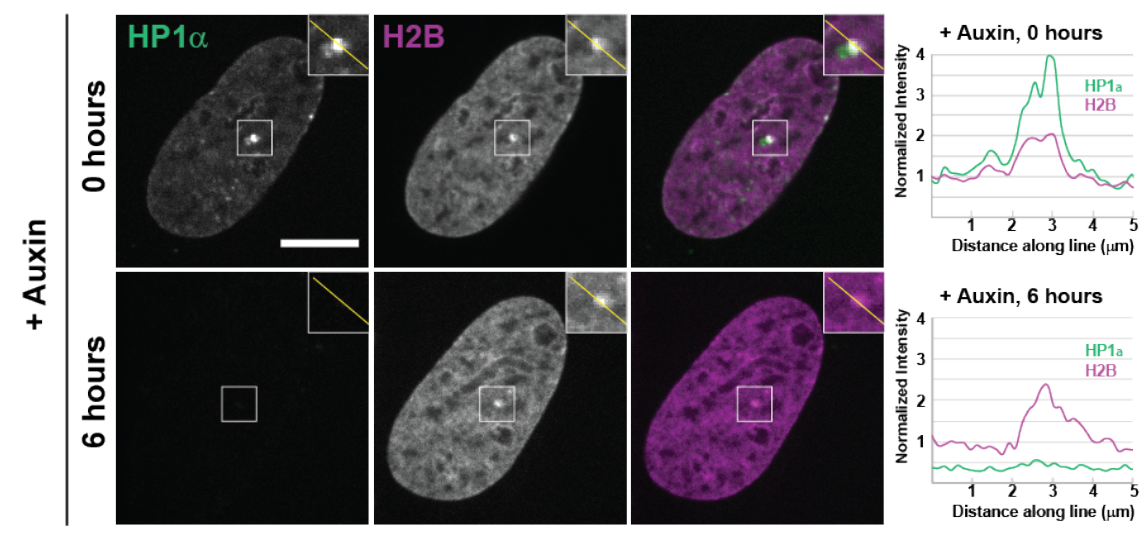

B
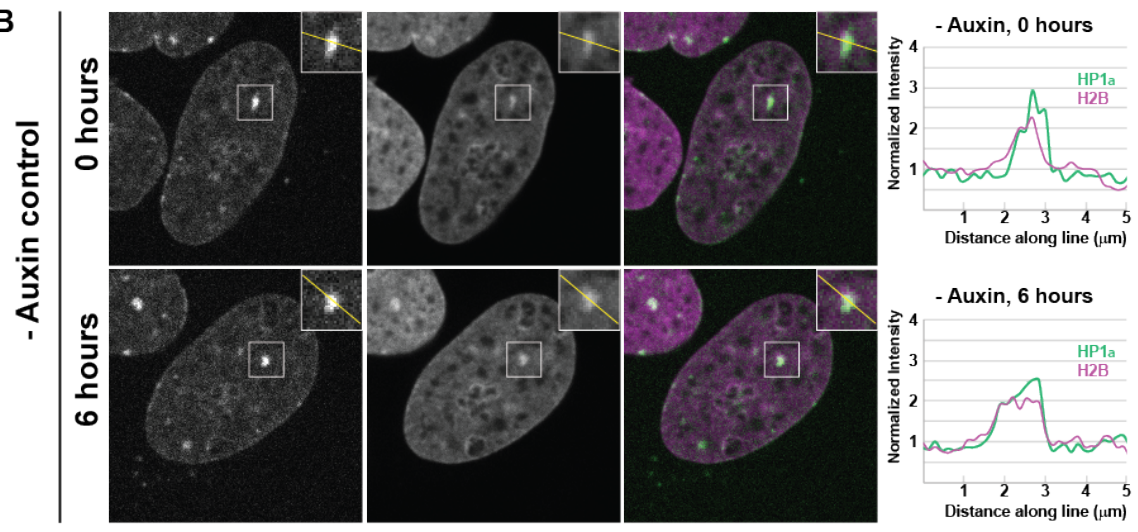

C

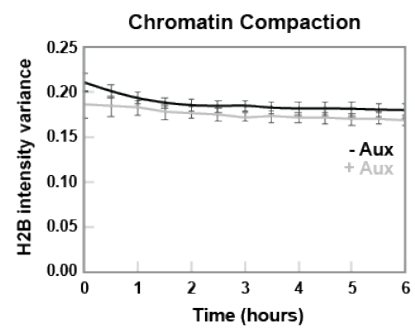

D

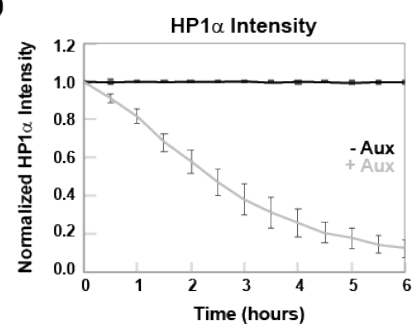

E

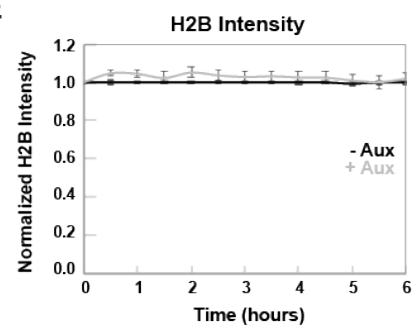

Figure 1 - figure supplement 1 . Local chromatin compaction remains after HP1a degradation.

(A, B) Example images and line scans of HP1a-AID-sfGFP and histone 2B (H2B-mCherry) before and after addition of $(A)+$ auxin or $(B)$ - auxin control. Scale bar $=10 \mu \mathrm{m}$. Graphs of average (C) chromatin compaction, (D) HP1a-AID-sfGFP intensity, and (E) H2B intensity for control - auxin (black line) and HP1 $\alpha$ degradation + auxin (gray line). $N=3$ trials, $n=20$ nuclei per trial. 


\section{HP1 $\alpha$ is a major mechanical component of the interphase nucleus that contributes to nuclear shape maintenance}

We hypothesized that HP1a could aid nuclear mechanics due to its association with heterochromatin. To test this hypothesis, we perform single-nucleus micromanipulation force measurements on untreated and auxin-induced HP1 1 -degraded nuclei. Micromanipulation is an extensional force measurement technique capable of separating chromatin- and lamin-based nuclear mechanics (Stephens et al., 2017). First, a single nucleus is isolated from a living cell following treatment with latrunculin A to depolymerize actin and local lysis applied via micropipette spray (Figure 2A). The isolated nucleus is then loaded between two micropipettes. One micropipette is moved to extend the nucleus, while the other micropipette's deflection, multiplied by the premeasured bending constant, measures force (Figure 2A). The force-extension relation is nonlinear, but can be decomposed into two linear slopes which provide a nuclear spring constants $(\mathrm{nN} / \mu \mathrm{m})$ for the short-extension regime $(<3 \mu \mathrm{m})$ quantifying chromatin-based stiffness and a long-extension regime $(>3 \mu \mathrm{m})$ quantifying lamin-based strain stiffening ((Stephens et al., 2019b, 2018, 2017); example Figure 2B).

Micromanipulation force measurements reveal that degradation of HP1a affects nuclear mechanics. Parental unmodified U2OS cells, control or auxin treated, show no change in the chromatin-based nuclear spring constant ( 0.35 vs. $0.34+/-0.06 \mathrm{nN} / \mu \mathrm{m}$, Figure $2 \mathrm{C})$. This suggests that auxin alone does not alter nuclear mechanics. HP1 $\alpha$-AID-sfGFP cells were imaged before nucleus isolation to verify presence or absence of HP1 $\alpha$ via sfGFP reporter. Auxin-induced HP1 $\alpha$ degradation resulted in a $45 \%$ decrease in short-extension chromatin-based nuclear stiffness ( 0.40 vs $0.22+/-0.03 \mathrm{nN} / \mu \mathrm{m}$, Figure 2D). However, long-extension strain stiffening remained relatively unchanged (example Figure 2B; Figure 2 - figure supplement $1 \mathrm{~A}$ ) in agreement with the observation of no decrease in lamin A/C or B1 levels (Figure 2 - figure supplement 1, B and C). This data indicates that HP1 $\alpha$ contributes to chromatin mechanics of the cell nucleus.

Previous work has shown that nuclear softening due to perturbations of chromatin and its mechanics, particularly the loss of heterochromatin, can induce abnormal nuclear morphology (Stephens et al., 2019b, 2018). Consistent with these prior findings, we quantified nuclear shape in HP1 $\alpha$-degraded cells and found that they displayed a significant increase in abnormal nuclei, from $10+/-1 \%$ in untreated cells to $22+/-5 \%$ upon HP1a loss, as quantified by nuclei below a solidity threshold (solidity < 0.97 , Figure $2 \mathrm{G}$ ). Another way to quantify shape is average nuclear curvature, which increases when the nucleus deviates from its normal elliptical shape (see Materials and Methods). Tracking average nuclear curvature of single nuclei over time post auxin treatment reveals a significant increase in nuclear curvature during interphase ( $0 \%$ control vs $36 \%$ HP1a-degraded single nuclei increase curvature by 0.05 , Figure 2 - figure supplement 2), coincident with HP1a loss and decreased nuclear stiffness (4 hour auxin, Figure 2). These data establish that HP1 $\alpha$ degradation results in nuclear softening and abnormal nuclear morphology. 


\section{HP1 $\alpha$ and histone methylation contribute independently to nuclear mechanics and morphology}

It is unclear exactly how the different components of heterochromatin work together to define its structure and function and how dependent they are on one another. For example, studies using genetic knockouts (Bosch-Presegué et al., 2017) and long-term depletion RNAi studies of heterochromatic components (Frescas et al., 2008; Hahn et al., 2013; Shumaker et al., 2006) have reported that HP1a not only binds to methylated histones, but also aids in histone methylation establishment and maintenance (Jacobs and Khorasanizadeh, 2002; Nielsen et al., 2002; Schotta, 2002). To determine if HP1a simply alters levels of methylated histones to affect nuclear mechanics and morphology, we measured levels of the constitutive heterochromatin mark $\mathrm{H} 3 \mathrm{~K} 9 \mathrm{me}^{2,3}$. Upon acute degradation of HP1a, H3K9me ${ }^{2,3}$ levels did not change significantly (Figure 2, E and F). Thus, while reduction of HP1a levels affects nuclear mechanics and morphology, it does not cause significant changes in histone methylation (Figure 2, E and F).

Previous reports have shown that increased histone methylation stiffens the nucleus (Stephens et al., 2019b, 2018, 2017). Cells were treated with the broad histone demethylase inhibitor methylstat, which increases $\mathrm{H} 3 \mathrm{~K} 9$ methylation approximately three-fold over its normal levels in the HP1a-AID-sfGFP cell line (Figure 2, E and F). Micromanipulation force experiments with HP1a-AID-sfGFP cells treated with $1 \mu \mathrm{M}$ methylstat for 48 hours measured a stiffer chromatinbased nuclear spring constant (Figure 2D), similar to previously reported experiments on different cell lines (Stephens et al., 2018). Increased broad histone methylation via methylstat did not significantly increase HP1a-AID-sfGFP levels. Thus, chromatin-based nuclear mechanics can be modulated by changing either HP1a levels or methylated histone levels.

We reasoned that elevating levels of methylated histone in HP1a-degraded nuclei would reveal the relative contributions of histone methylation and HP1 $\alpha$ to nuclear mechanics and shape. If chromatin mechanics is dictated entirely by $\mathrm{HP} 1 \alpha$, increasing histone methylation in auxin-treated cells should not change nuclear mechanics; in that case, the nuclear spring constant should match that of the HP1a-degraded cells. Alternatively, if the methylation state of histones

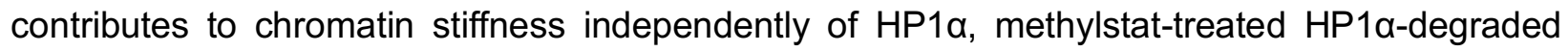
nuclei will have a larger spring constant than HP1a-degraded nuclei, and may display rescued nuclear shape.

Experiments were consistent with the second scenario, where increasing histone methylation levels in HP1a-degraded cells resulted in rescued nuclear mechanics and shape. Micromanipulation force measurements revealed a larger nuclear spring constant for HP1 $\alpha$ degraded nuclei with increased histone methylation as compared to HP1 1 -degraded with normal levels of methylation, returning to a spring constant similar to wild-type levels (auxin 0.22 vs. auxin+methylstat $0.33+/-0.03 \mathrm{nN} / \mu \mathrm{m}$, Figure 2D). Alternatively, compared to normal levels of $\mathrm{HP} 1 \alpha$ with increased histone methylation, loss of HP1 $\alpha$ and increased histone methylation resulted in a decreased nuclear spring constant (auxin+methylstat 0.33 vs. mehtylstat $0.56+/$ $0.03 \mathrm{nN} / \mu \mathrm{m}$, Figure 2D). Strain stiffening in the lamin-dependent regime remained similar across 
all treatments (Figure 2 - figure supplement 1). Consistent with the mechanical measurements, methylstat treatment partially rescues abnormal morphology associated with HP1a degradation (Figure 2G). Altogether, these results suggest that HP1 $\alpha$ and methylated histone levels both contribute to chromatin-based nuclear mechanics and morphology. Moreover, the approximately additive nature of the changes in nuclear stiffness, along with the lack of interdependence between levels HP1 $\alpha$ and histone methylation, suggest that these mechanisms contribute to mechanics independently.

Figure 2. HP1a is a mechanical component of the nucleus controlling nuclear shape, separately from histone methylation.

(A) Example images of a single isolated nucleus via transmitted light and HP1a-AID-sfGFP fluorescence and single nucleus micromanipulation force extension measurement experiment. The pull pipette extends the nucleus while the bending of a premeasured force pipette provides force measurement. Scale bar $=10 \mu \mathrm{m}$. (B) Example traces of micromanipulation force-extension for control (black) and auxin-induced degradation of HP1a (orange) provide a measure of nuclear spring constant from the slope (dotted lines). Initial slope provides chromatin-based nuclear spring constant while the second slope provides the lamin -based strain stiffening nuclear spring constant. ( $C$ and $D)$ Graphs of average and single chromatin-based nuclear spring constant for (C) parental cell line control and 4-6 hour auxin treated and (D) HP1a-AID-sfGFP with and without auxin and/or methylstat treatment. $n=11$ - 18 nuclei each. (E) Example images of cells treated with and without auxin and/or methylstat. (F) Quantified relative fluorescence of HP1a-AID-sfGFP and heterochromatin marker $\mathrm{H} 3 \mathrm{~K} 9 \mathrm{me}^{2,3}$. (G) Quantified abnormal nuclear morphology determined as solidity value less than 0.97 . Another way to quantify abnormal nulcear morphology is via average nuclear curvature reported in Flgure 2 - figure supplement 2. $N=3$ experiments each (shown as black dots) each consisting of $n>100$ control, $n>100$ auxin, $n>30$ methylstat, and $n>78$ auxin methylstat. $P$ values reported as ${ }^{*}<0.05,{ }^{* *}<0.01,{ }^{* * *}<0.001$. Error bars represent standard error. 
A

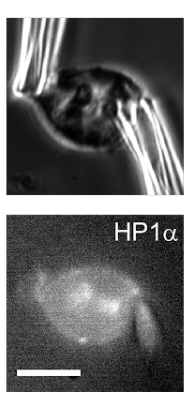

C

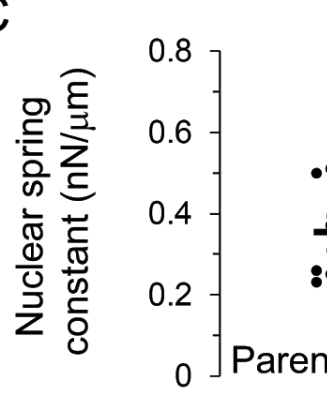

B
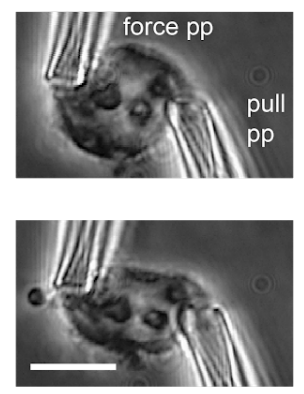

$p>0.05$

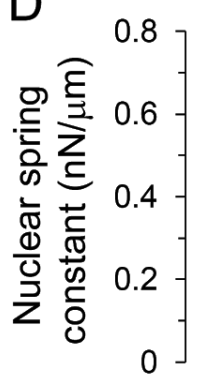

Auxin

(deg HP1 $\alpha$ )

Methylstat

(his. methylation)

$\mathrm{E}$

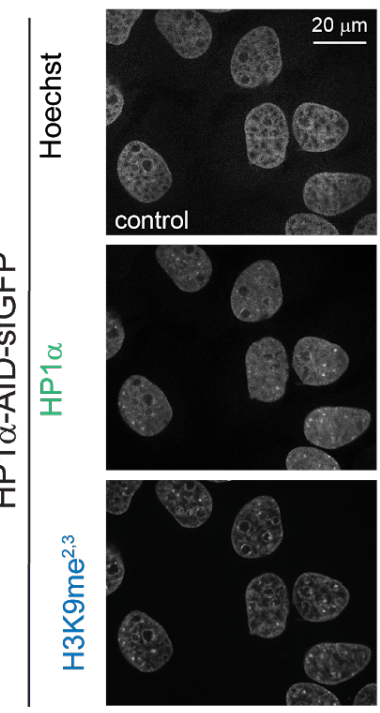

$\mathrm{F}$

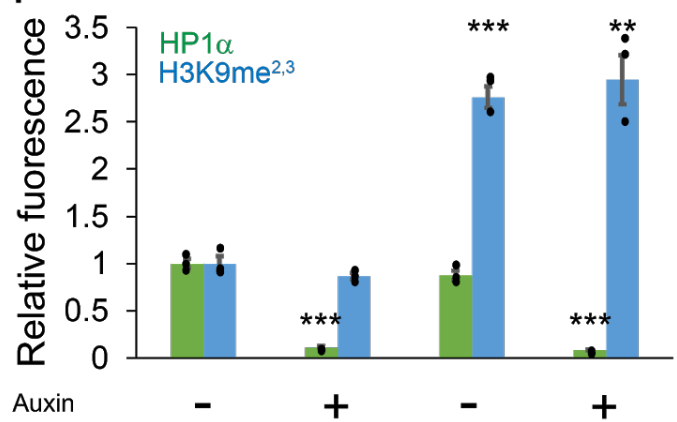

(deg HP1 $\alpha$ )

Methylstat

(his. methylation)
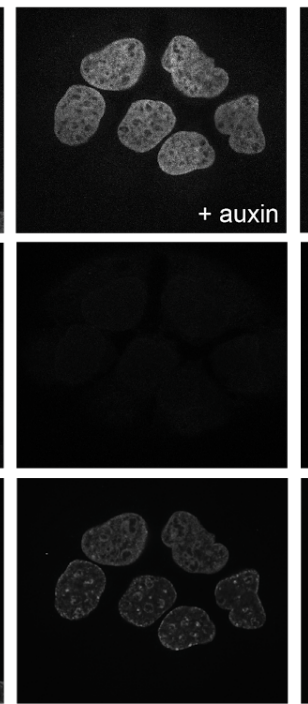

G
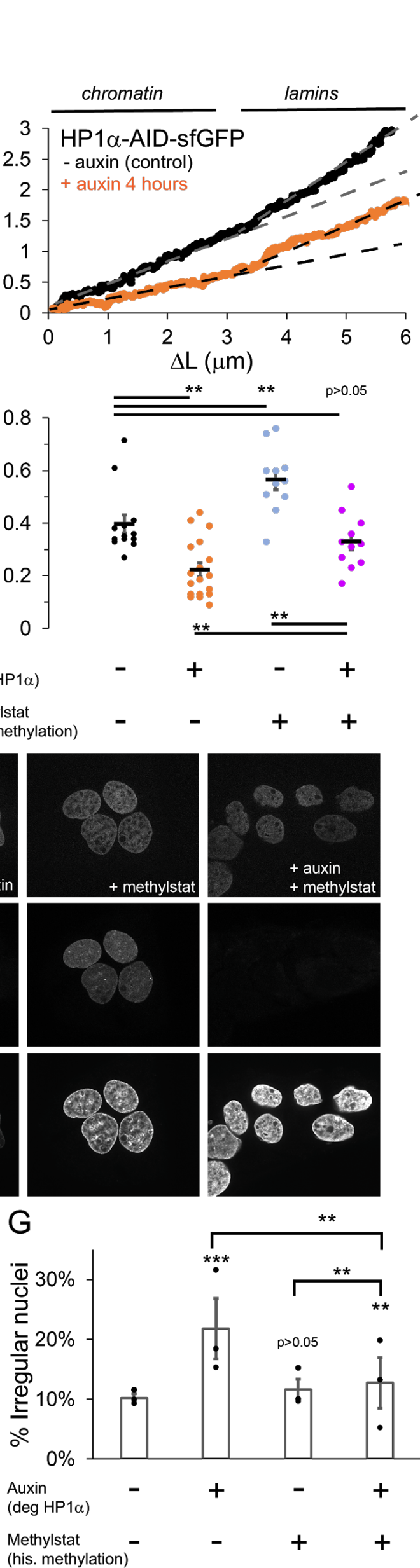
bioRxiv preprint doi: https://doi.org/10.1101/2020.10.09.331900; this version posted October 9, 2020. The copyright holder for this preprint (which was not certified by peer review) is the author/funder, who has granted bioRxiv a license to display the preprint in perpetuity. It is made available under aCC-BY-NC-ND 4.0 International license.

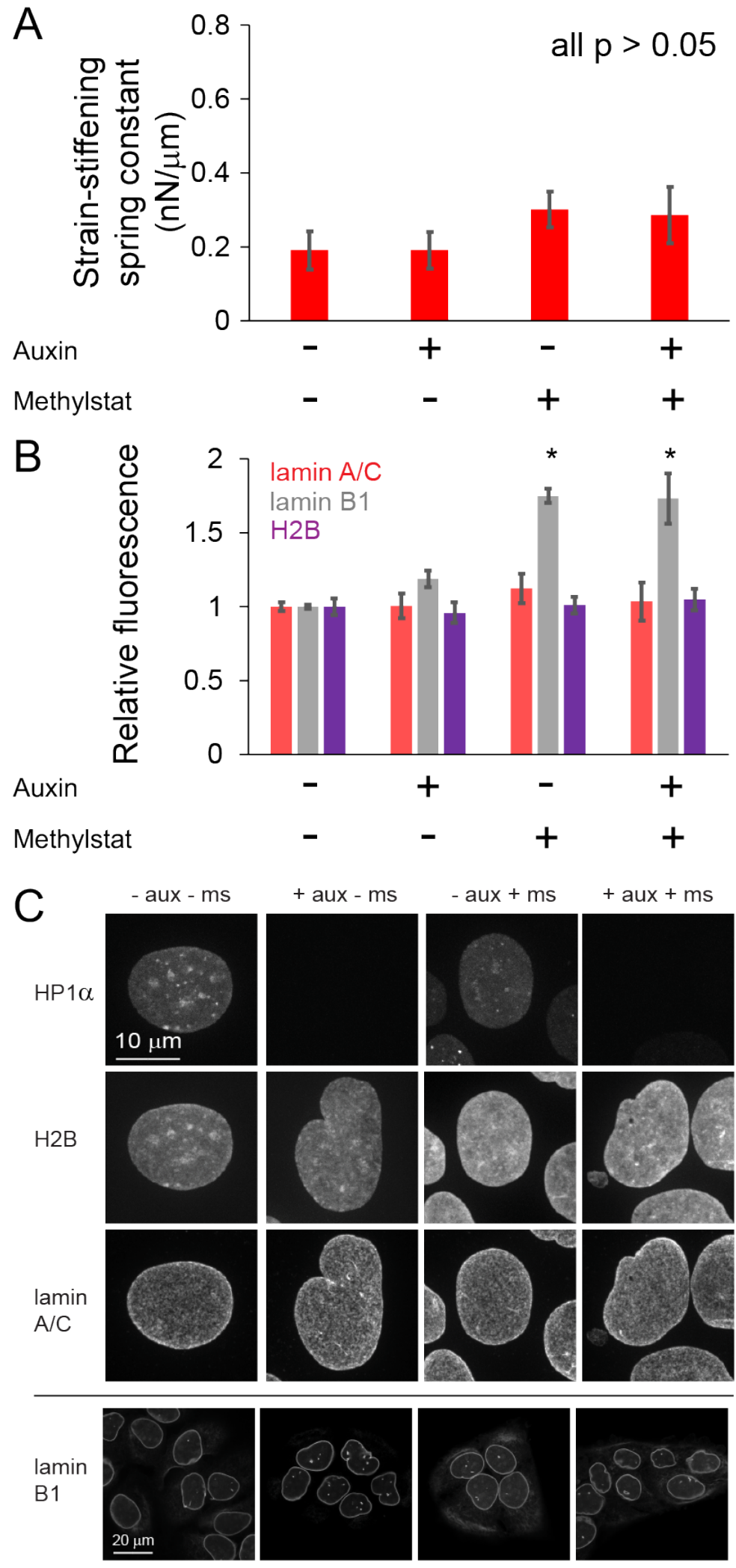


Figure 2 - figure supplement 1. Lamin mechanics and levels do not change upon degradation of HP1a.

(A) Strain-stiffening nuclear spring constant measured as the long extension force-extension slope minus the short extension (chromatin-based) force-extension slope. $\mathrm{n}>9$ for each condition. This data was taken simultaneously with force data presented in Figure 2. (B) Graph of the average of relative fluorescence for lamin $A / C$ and lamin B1 levels upon auxin and/or methylstat treatment. For lamin $\mathrm{A} / \mathrm{C}$ and $\mathrm{H} 2 \mathrm{~B} \mathrm{~N}=3$ experiments where $\mathrm{n}>25$ nuclei each. For lamin B1 $\mathrm{N}=3$ experiments for each condition consisting of $n>100$ control, $n>100$ auxin, $n>$ 30 methylstat, and $n>78$ auxin methylstat, this data was acquired along with those presented in Figure 2, E and F. P values reported as ${ }^{*}<0.05,{ }^{* *}<0.01,{ }^{* * *}<0.001$. (C ) Example images. Error bars represent standard error.

Figure 2 - figure supplement 2 . Increased nuclear curvature onsets coincident with HP1a loss during interphase.

Graphs of average nuclear curvature for single cells over time for U2OS HP1a-AID-sfGFP expressing H2B-miRFP (A) without auxin and (B) with auxin added. Percentage of nuclei that drastically ( 0.05 curvature) and stably (more than 3 time points) changed shape are denoted in the bottom-right hand corner. Histograms of (C)untreated (ctrl) and (D) auxin-treated (aux) nuclear curvature at time $1,5,12$ hours. The dotted line denotes abnormally shaped nuclei $(>0.175)$ and upper right percentages of abnormal nuclei. (E) Average population nuclear curvature over time with (gray) and without (black) the addition of auxin at time zero. Error bars represent standard error, $n=42$ nuclei each condition. (F) Example images of single nucleus treated with auxin over time shows loss of normal elliptical morphology during interphase. P values reported as * $<0.05$, ${ }^{* *}<0.01,{ }^{* * *}<0.001$ 

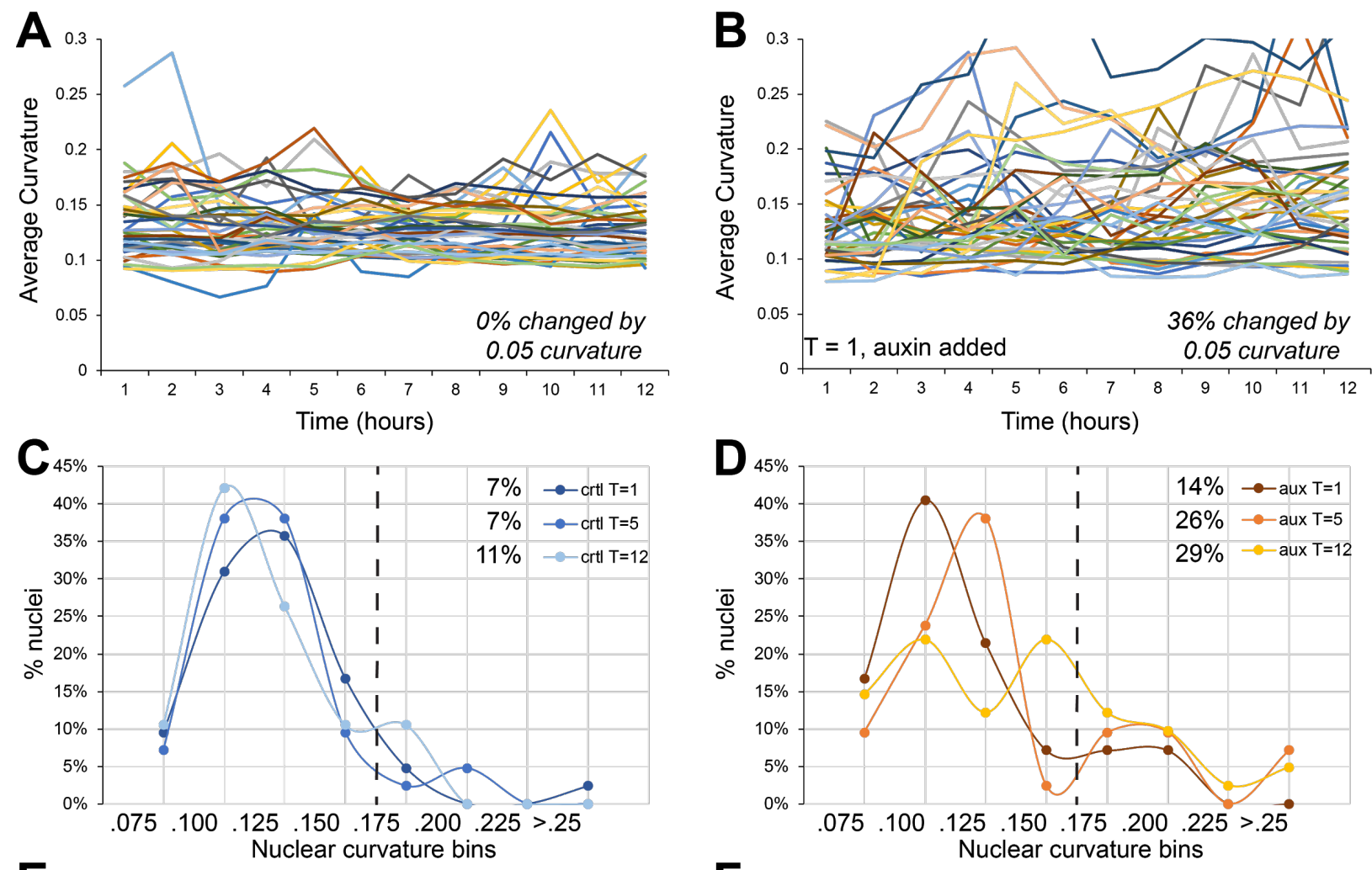

$\mathbf{E}$
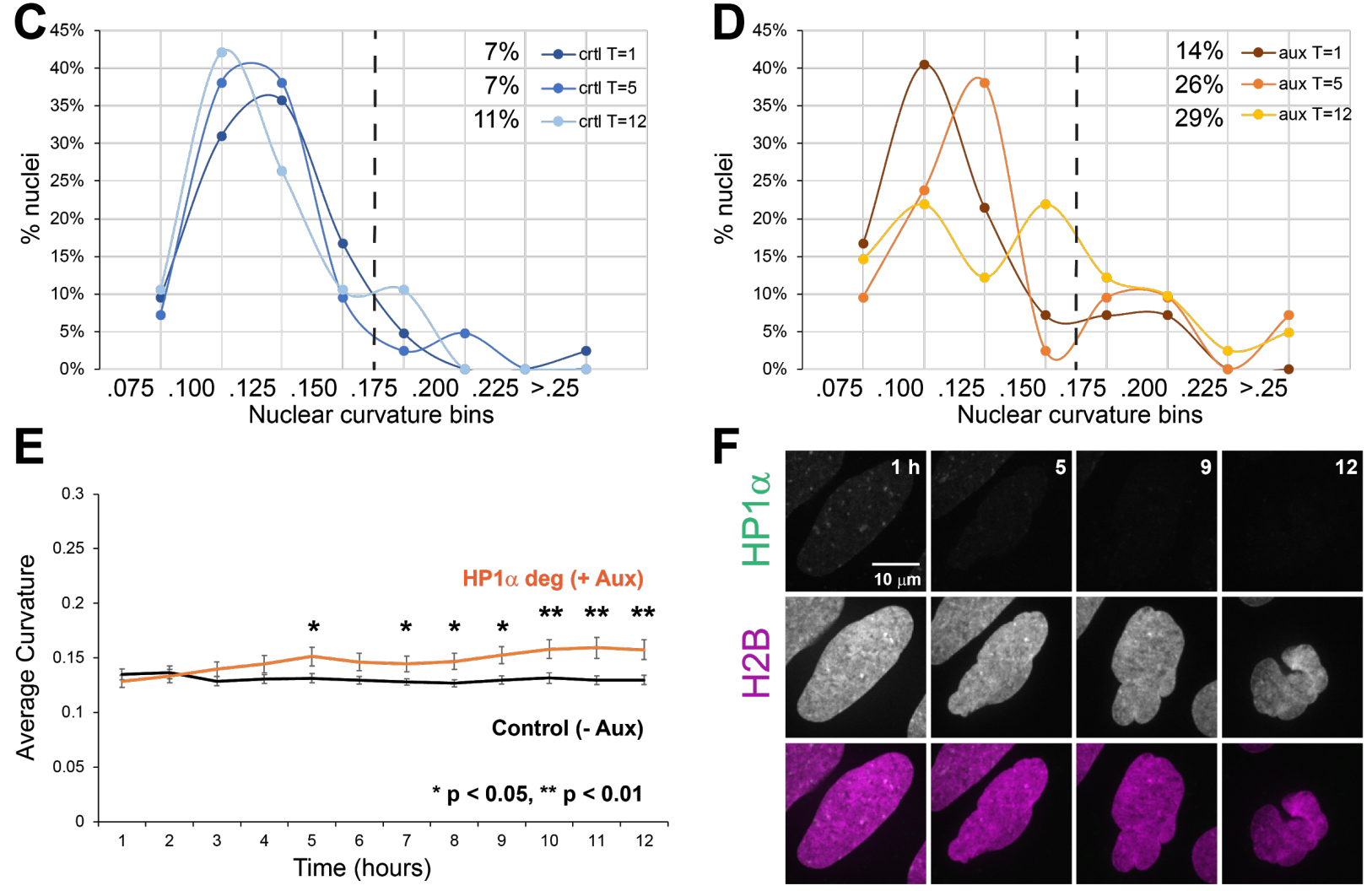


\section{Maintenance of nuclear morphology depends on HP1 $\alpha$ dimerization}

HP1a forms a homodimer that can bridge strands of chromatin by binding two $\mathrm{H} 3 \mathrm{~K} 9 \mathrm{me}^{2,3}$ marks on different nucleosomes through its chromodomain (Jacobs and Khorasanizadeh, 2002; Machida et al., 2018; Nielsen et al., 2002) or two strands of DNA through a positively charged KRK patch in the hinge (Larson et al., 2017). We reasoned that the role of HP1a in determining nuclear shape and mechanics, independent of histone methylation levels, might be due to its ability to physically crosslink chromatin strands. This linking ability would be dependent on HP1 $\alpha$ dimerization, which can be disrupted with a point mutant, HP1 $\alpha^{1165 E}$ (Brasher et al., 2000; Thiru et al., 2004). To determine if dimerization is key to its mechanical and morphological contributions in vivo, we asked whether a non-dimerizing mutant $\left(\mathrm{HP} 1 \alpha^{1165 \mathrm{E}}\right)$ could rescue nuclear morphology when the endogenous protein was degraded.

HP1 $\alpha$-AID-sfGFP cells were infected with lentivirus to stably express either exogenous HP1 $\alpha^{\text {WT }}$ mCherry (positive control) or HP1 $1 \alpha^{1165 E}$-mCherry (dimer mutant, (Brasher et al., 2000)) under an SFFV promoter. Two days post-infection, these cells stably expressing HP1 $\alpha^{\mathrm{WT}}$-mCherry or $\mathrm{HP} 1 \alpha^{1165 E}$-mCherry were treated with $1 \mathrm{mM}$ auxin for 16 hours to degrade the endogenous HP1 $\alpha-$ AID-sfGFP and assess the ability of the rescue construct to maintain and recover normal nuclear shape. These cells were fixed and immunostained for lamin $A / C$, and then the shape of the nucleus was quantified using the Kappa plugin for FIJI to calculate the average curvature of each nucleus (Schindelin et al., 2012).

We first measured nuclear curvature in control and auxin-treated HP1a-AID-sfGFP cells to determine normal and abnormal nuclear curvature, respectively. Similar to our previous measurements above (Figure 2 and Figure 2 - figure supplement 2), control cells showed a lower average curvature compared to higher curvature in HP1a-degraded nuclei (Figure 3). Expression

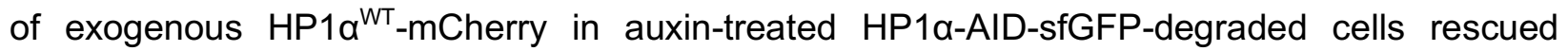
nuclear morphology to near wild-type levels ( $p>0.05$, - aux vs. +aux HP1 $\alpha^{\mathrm{WT}}$-mCherry). However, the dimer mutant HP1 $\alpha^{165 E}$-mCherry did not rescue nuclear morphology in auxin-treated cells (Figure 3; control, 0.116 +/- 0.003; HP1a-degraded with no rescue, 0.142 +/- 0.001; HP1adegraded with HP1 $\alpha^{\text {WT }}$ rescue, $0.123+/-0.002$; HP1 1 -degraded with HP1 $\alpha^{1165 E}$ rescue, $0.151+/-$ 0.003 ). We observed that a subset of nuclei had abnormal nuclear shape (curvature greater than one standard deviation above control). Using this metric, cells in which HP1a-AID-sfGFP was degraded displayed a higher level of abnormally shaped nuclei compared to control (30\% vs. $11 \%$, Figure $3 \mathrm{C}$ ). Expression of $\mathrm{HP} 1 \alpha^{\mathrm{WT}}$-mCherry recovered WT levels (13\% abnormal), while expression of $\mathrm{HP} 1 \alpha^{1165 E}$-mCherry did not, leaving many abnormally shaped nuclei $(31 \%)$. Together, these results indicate that HP1 $\alpha$ dimerization is essential to its function in nuclear morphology and indicates that bridging or crosslinking of chromatin fibers is important in determining nuclear shape. 

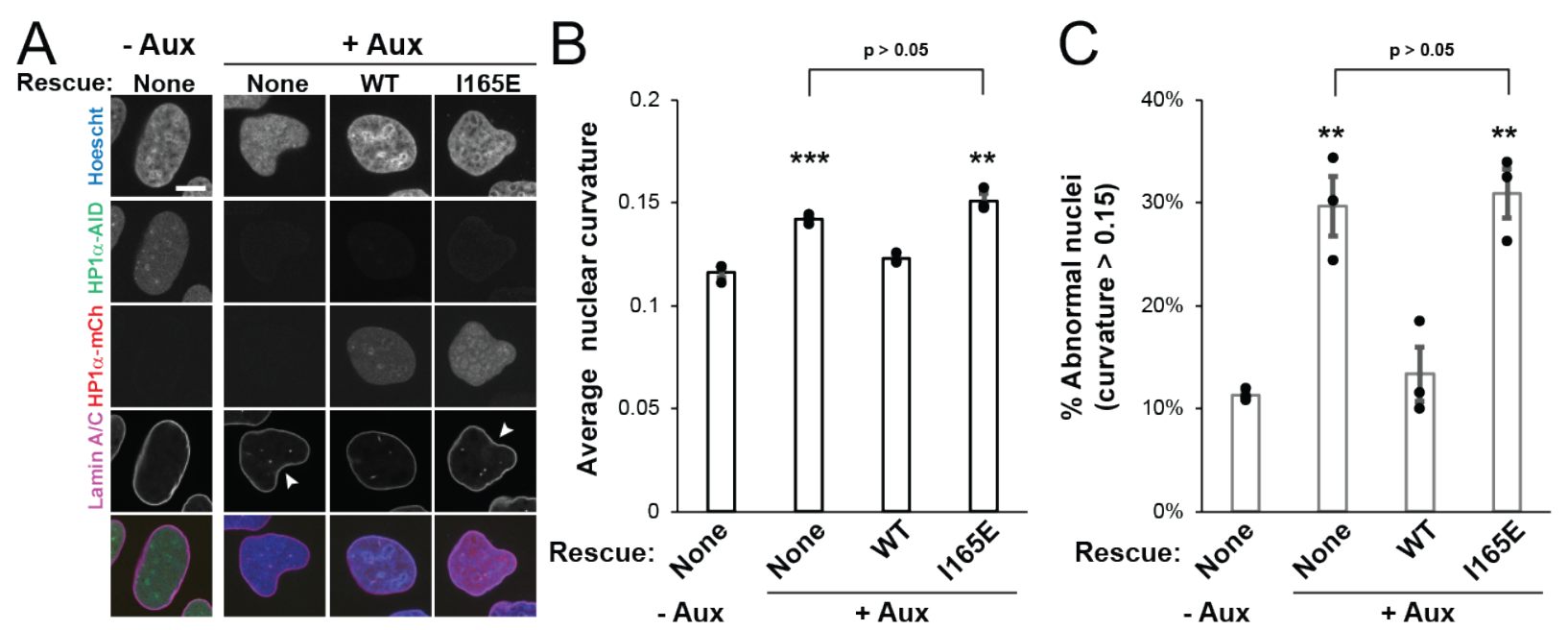

Figure 3. HP1a dimerization is essential for maintenance of nuclear shape.

(A) Example images of HP1a-AID-sfGFP cells control and auxin treated with and without exogenous HP1 1 wild-type (WT) or dimer mutant (I165E) rescue constructs tagged with mCherry. Scale bar $=10 \mu \mathrm{m}$. (B) Graph of average nuclear curvature measurements of the different trials as black dots. (C) Graph of percentage of abnormally shaped nuclei, determined as greater than 0.15 curvature, which is the average untreated nucleus plus the standard deviation. 3 experiments for each condition (denoted as black dots) consisting $n=25-50$ nuclei each. Error bars represent standard error.

\section{Simulations of nuclear mechanics modulating chromatin crosslinking recapitulate experimental degradation of $\mathrm{HP} 1 \alpha$}

To assess the role of HP1a in chromatin-based nuclear mechanical response, we performed Brownian dynamics simulations using a previously developed shell-polymer model (Banigan et al., 2017; Stephens et al., 2017). In these simulations, chromatin is modeled as a crosslinked polymer that is physically linked to a peripheral polymeric lamin shell that encapsulates the polymer chromatin (see Materials and Methods). In this model, each chromatin bead is $0.57 \mu \mathrm{m}$ in diameter and represents a few $\mathrm{Mb}$ of the genome. This coarse-grained model can capture the effects of alterations to histone modifications through the polymer spring constant and perturbations to lamin $\mathrm{A} / \mathrm{C}$ through the lamin spring constant (pink data points in Figure $4 \mathrm{~A}$ and C; (Stephens et al., 2017)). In particular, varying the polymer spring constant models alterations to chromatin compaction via histone modifications (Stephens et al., 2017); the short-extension nuclear force response is suppressed as the polymer spring constant is decreased, but the longextension response is largely unchanged (Figure 4 - figure supplement $1 \mathrm{~A}$ ).

However, acute depletion of HP1a does not alter histone methylation state or lamin expression levels (Figure 2, E and F; Figure 2 - figure supplement 2), so we sought to identify a distinct physical role for HP1 $\alpha$ within this framework. We hypothesized that HP1 $\alpha$ might instead govern mechanics either by tethering heterochromatin to the lamina via proteins such as PRR14 (Poleshko et al., 2013) and LBR (Polioudaki et al., 2001; Ye et al., 1997), or by binding and 
bridging nucleosomes (Azzaz et al., 2014; Canzio et al., 2011; Erdel et al., 2020; Machida et al., 2018). Thus, we explored whether HP1 a might impact nuclear mechanical response by forming chromatin-chromatin crosslinks or by forming chromatin-lamina linkages.

We first investigated whether HP1 $1 \alpha$ might govern mechanical response by acting as a chromatinlamina linker. In simulations, we varied the frequency of linkages between the chromatin and the lamina from zero up to $\sim 50 \%$ of the chromatin subunits that reside near the shell. We found that the frequency of chromatin-lamina linkages affects the two-regime force response of the model nucleus (Figure 4A). The spring constants quantifying both the short- and long-extension force responses decrease as the number of chromatin-lamina linkages is decreased (Figure 4B). With fewer chromatin-lamina linkages, the mechanical coupling between the nuclear periphery and the interior is lost, which suppresses short-extension rigidity; simultaneously, the loss of these linkages uncouples the lamina from the stiff chromatin interior, which also decreases the longextension stiffness. This result contrasts with measurements from the micromanipulation experiments (Figure 2D; Figure 2, Supplement Figure 1), which show that the short-extension spring constant, but not the long-extension spring constant, decreases after HP1a degradation. Thus, we conclude that the mechanical contributions of HP1 $\alpha$ arise from an alternative structural function.

We therefore investigated the effects of varying the levels of chromatin-chromatin crosslinkers in the simulation model. We varied crosslinking frequency from zero up to about one in three subunits crosslinked, above which the chromatin polymer is percolated network and therefore solid-like. We found that the level of crosslinking markedly alters the force-strain (Figure 4C); increasing crosslinking stiffens the nucleus. However, in contrast to chromatin-lamina linkages, crosslinks govern stiffness of only the short-extension force response (Figure 4D). This is a signature of their specific effect in resisting deformations of the chromatin interior. These qualitative trends agree with the measurements from micromanipulation experiments (Figure 2, $B$ and D). The simulation data also includes points that are in reasonable quantitative agreement with the experiments. Simulations show that the same effect is difficult to achieve by simply varying the stiffness of the crosslinks. Above a relatively small threshold, the short-extension nuclear spring constant is insensitive to the stiffness of the crosslinker (Figure 4 - figure supplement 1B). These results are consistent with a model in which the HP1 $\alpha^{1165 E}$ mutant abolishes crosslinking (Machida et al., 2018) and thus decreases the short-extension nuclear spring constant (Figure 2, B and D), which may generate abnormal nuclear morphology. Altogether, the simulations support the conclusion that HP1 $\alpha$ contributes to nuclear mechanical response by acting as a chromatin-chromatin crosslinking element. 

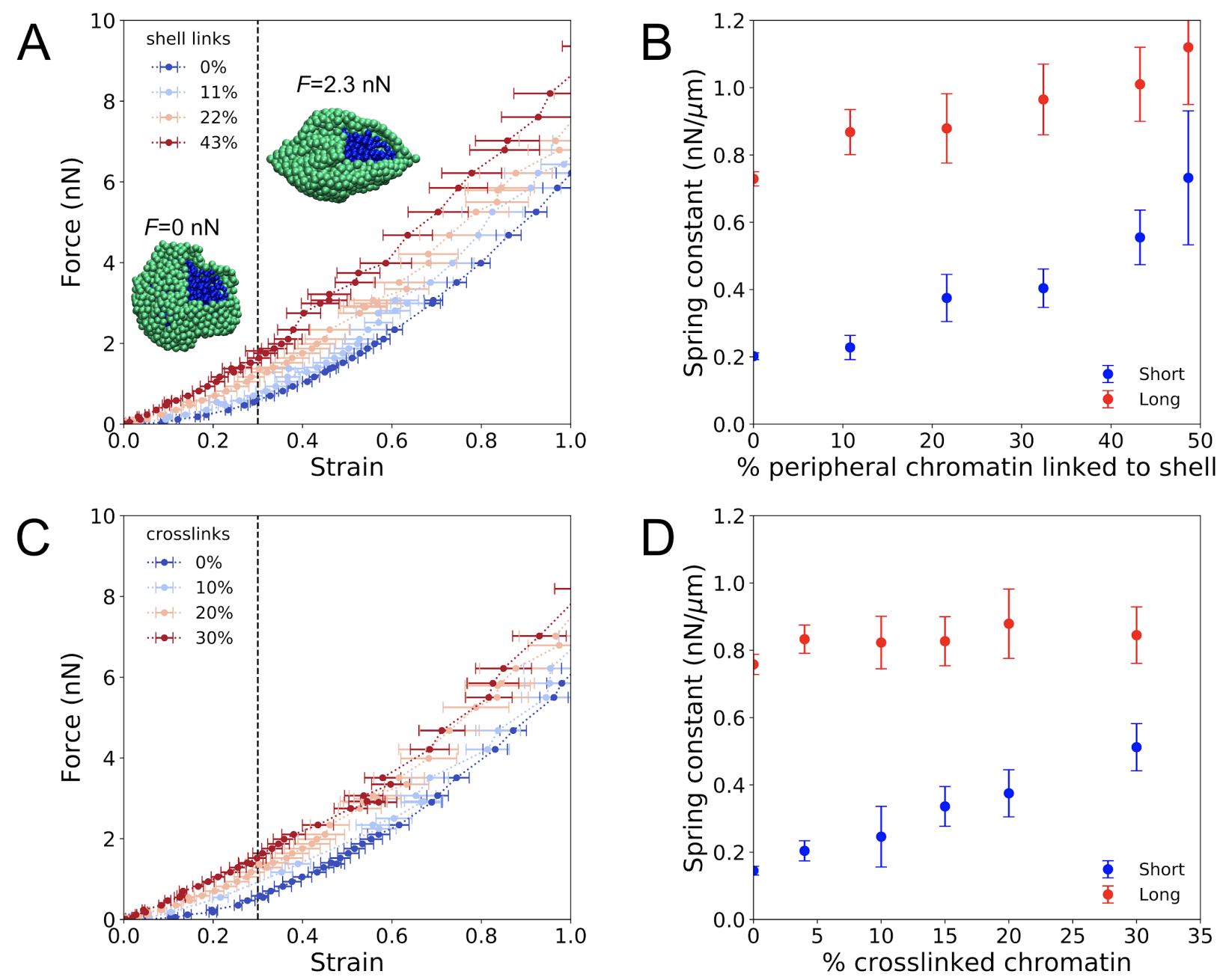

Figure 4. Simulations of nuclear mechanical response support a model with HP1a as a chromatin-chromatin crosslinker.

(A) Force-strain relationship for simulated nuclei with various levels of chromatin-lamina (shell) linkages. Colors indicate different percentages of chromatin segments linked to the lamina. Insets: Snapshots of simulations with a portion of the lamina (green) removed to reveal the interior chromatin (blue) for two different applied stretching forces, $F$. (B) Spring constants for short- and long-extension regimes for simulations with various levels of chromatin-lamina crosslinks, quantified by percentage of peripheral chromatin subunits linked to the shell (blue and red, respectively). (C) Force-strain relation for simulated nuclei with various levels of chromatinchromatin crosslinks. (D) Spring constants for short- and long-extension with varying levels of chromatin-chromatin crosslinks (blue and red). Vertical dashed lines in (A) and (C) separate the short-extension and long-extension regimes. Each force-strain data point is an average from $n \geq$ 11 simulations. Short and long spring constants are each computed from $n_{\text {short }} \geq 13$ and 7 and $\mathrm{n}_{\text {long }} \geq 15$ and 14 force-extension data points for $(B)$ and (D), respectively. Error bars show standard error of the mean in all plots. 

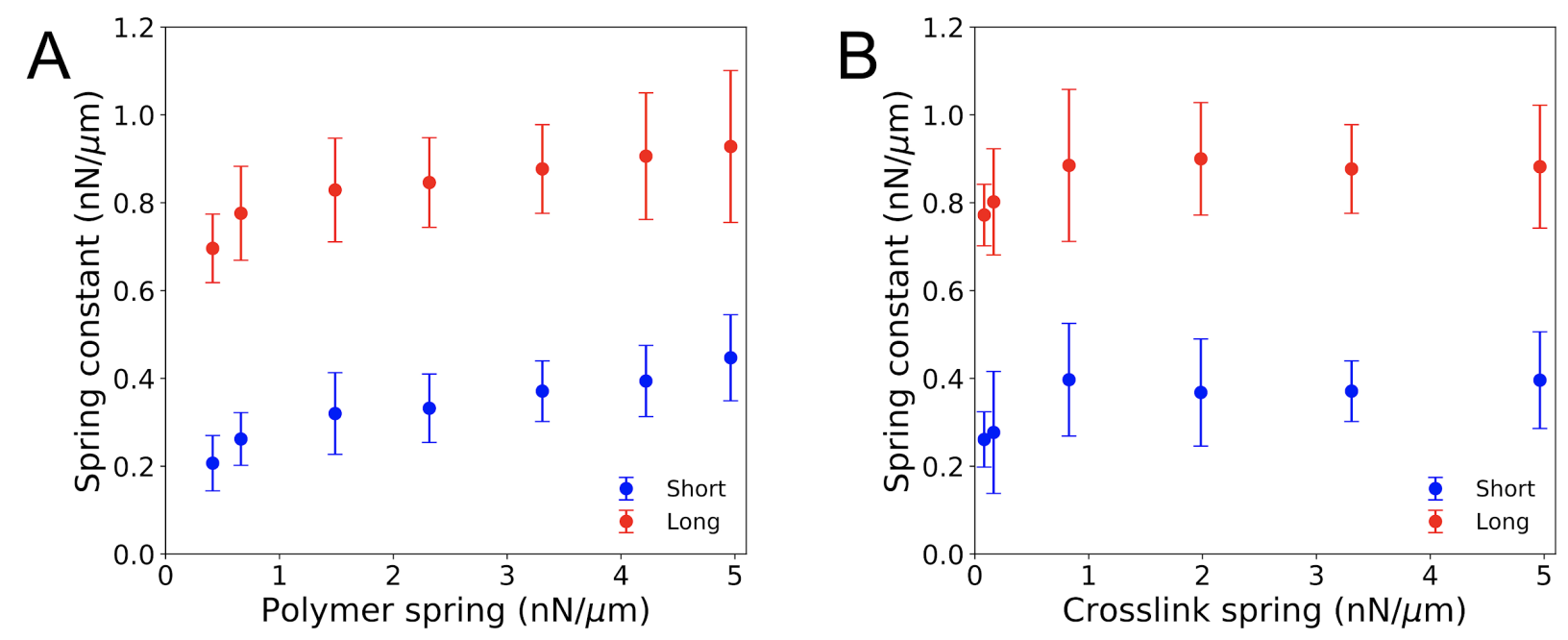

Figure 4 - figure supplement 1 . Spring constants measured in simulations with different polymer springs or crosslink springs.

Spring constants for short- and long-extension regimes (blue and red, respectively) for simulations with various (A) chromatin (polymer) spring constants and (B) crosslink spring constants. Spring constants in (A) and (B) are each computed from $n_{\text {short }} \geq 9$ and 7 and $n_{\text {long }} \geq 8$ and 8 force-extension data points, respectively. Each force-extension data point is an average from $n \geq 11$ simulations. Error bars show standard error of the mean in all plots. 


\section{HP1a provides mechanical strength to mitotic chromosomes}

Given HP1a's mechanical role in chromatin-based nuclear mechanics, we hypothesized that HP1 1 could also contribute to mitotic chromosome mechanics. Most HP1 $\alpha$ is removed from chromosomes during prophase by phosphorylation of H3S10, which is known to disrupt HP1aH3K9me ${ }^{2,3}$ binding (Fischle et al., 2005; Hirota et al., 2005). However, some HP1a binding is maintained throughout mitosis (Serrano et al., 2009), suggesting a possible role for HP1a in mitotic chromosome mechanics.

We used fluorescence imaging and micropipette micromanipulation methods (Biggs et al., 2019; Sun et al., 2018) to assay the presence of HP1a-AID-sfGFP in prometaphase cells (identified by their round shape) and mitotic chromosomes without or with auxin treatment for four hours to

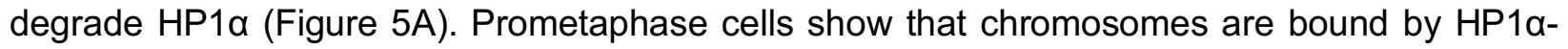
AID-sfGFP, with additional cytoplasmic protein giving a diffuse signal. Both cytoplasmic and chromosomal HP1a-AID-sfGFP signals nearly completely disappear upon auxin-induced degradation (Figure 5A). The mitotic chromosome bundle can be isolated from the cell via gentle lysis and capture (Figure 5, B and C). Fluorescence imaging of an isolated mitotic chromosome bundle showed that HP1 $\alpha$ remains bound to isolated mitotic chromosomes (Figure 5C). Thus, we confirmed that endogenous HP1 1 -AID-sfGFP is associated with mitotic chromosomes and degraded after four hours of auxin treatment.

The mechanical role of HP1a in mitotic chromosomes was investigated by micromanipulation force measurement. The isolated bundle of chromosomes was held by one micropipette while two additional micropipettes were used to capture and isolate a single chromosome (Figure 5B). The single mitotic chromosome is then extended with the stiff "pull" pipette, while deflection of the other, much less stiff "force" pipette provides a force measurement, in the same manner as our experiments on interphase nuclei (Figure 5C). For each isolated chromosome, we calculated a force versus extension plot (Figure 5D). Because each of the 23 human chromosomes is a unique length, we calculate a length-independent measurement by extrapolating the force-extension slope to determine the 'doubling force'-the force at which the chromosome length would be doubled (i.e., force at $100 \%$ strain, Figure 5E). We find that depletion of HP1a reduced mitotic chromosome doubling force by approximately $40 \%$, from $310 \pm 60 \mathrm{pN}$ in control cells (spring constant $\sim 31 \mathrm{pN} / \mu \mathrm{m})$ to $170 \pm 10 \mathrm{pN}$ in auxin-treated cells $(\sim 18 \mathrm{pN} / \mu \mathrm{m})$ (Figure $5, \mathrm{~F}$ and $\mathrm{G}$ ), indicating that HP1 $\alpha$ significantly contributes to mitotic chromosome mechanics.

We next investigated whether histone methylation and the HP1 1 protein separately govern chromosome mechanics during mitosis, as they do during interphase. Increasing histone methylation via methylstat treatment has previously been shown to play a critical role in mechanical stiffness of mitotic chromosomes (Biggs et al., 2019). Furthermore, evidence exists for direct biochemical interactions between epigenetic marks on nucleosomes, independent of mark-reading proteins such as HP1a (Bilokapic et al., 2018; Zhiteneva et al., 2017). Thus, we aimed to determine whether histone methylation and HP1 $\alpha$ contribute independently to mitotic chromosome stiffness. 
We treated cells with the histone demethylase inhibitor methylstat to increase levels of methylated histones in cells with or without HP1a, controlled by the addition of auxin. Mitotic chromosomes isolated from cells treated with methylstat to increase methylated histone levels indeed show a significant, nearly $100 \%$ increase in doubling force from $310 \pm 60 \mathrm{pN}$ to $610 \pm 140 \mathrm{pN}(\sim 77 \mathrm{pN} / \mu \mathrm{m})$ (Figure 5, D and E), recapitulating previous results for HeLa cells (Biggs et al., 2019). Mitotic chromosomes isolated from cells treated with both methylstat to increase methylation and auxin to degrade HP1 $\alpha$ have a doubling force comparable to those treated with methylstat alone, $510 \pm$ $180 \mathrm{pN}(\sim 68 \mathrm{pN} / \mu \mathrm{m}, \mathrm{p}>0.05$, Figure 5, D and E). The data suggest that histone methylation stiffens mitotic chromosomes independently of HP1 $\alpha$ and thus has a dominant role in determining mitotic chromosome mechanics. At the same time, we emphasize that HP1 a clearly plays a major role in mitotic chromosome mechanics in wild-type cells.

HP1a depletion is known to lead to chromosomal instability, aberrant recombination, and metaphase bridges/lagging chromosomes (Chu et al., 2014). Therefore, HP1a's role in metaphase chromosome mechanics may have a functional role during mitosis. To test this, we measured the percentage of mitotic cells with chromosome misalignment in metaphase or anaphase bridges during chromosome segregation in control and 16-hour auxin-treated HP1 $\alpha$ degraded populations. HP1 1 depletion resulted in a significant increase in both metaphase misalignment, from $6 \%$ to $38 \%$, and mis-segregation as measured by anaphase bridges, from $12 \%$ to $46 \%$ (Figure 5, F - H). Thus, loss of HP1 1 disrupts chromosome mechanics or organization sufficiently to cause dysfunction in mitosis via chromosome misalignment and mis-segregation. 
Figure 5. HP1a is a mechanical component of the mitotic chromosome aiding proper segregation in mitosis.

(A) Representative live mitotic cells imaged via phase contrast and HP1a-AID-sfGFP fluorescent intensity across different treatments. The graph provides quantification of HP1 $\alpha$ fluorescence from the cells where chromosomes were isolated. Values calculated by measuring the cell's fluorescence minus the background fluorescence, normalized to the average intensity of the untreated cellular HP1 1 fluorescent intensity. Both auxin $(4 \mathrm{~h})$ and auxin $(4 \mathrm{~h})$ plus methylstat (48 h) are statistically significantly different from the control and methylstat treated cells. The control and methylstat treated cells were not statistically significantly different. (B) Example image of the steps to isolating a mitotic chromosome from a live cell using micropipettes.(C) Left panels show an example image of the endogenous HP1a-AID-sfGFP fluorescence of an isolated mitotic bundle outside of the lysed cell. Right panels show example images of a Force-Extension experiment. The right pipette pulls away from the left pipette, which stretches the chromosome and causes the left pipette to deflect. (D) Example traces of force-extension experiments for the different conditions. (E) Graph of average doubling force (100\% strain) in piconewtons for each condition, which is determined by slope of the force extension traces and the initial chromosomes length. For A-E $n=14$ for control and auxin treated, $n=8$ for methylstat and auxin methylstat treated. (F) Example images of abnormal mitotic segregation via anaphase bridge or nondisjunction. Graphs of percentage of mitotic cells from various phase displaying $(G)$ metaphase misalignment and $(H)$ anaphase/telophase missegregation via presence of anaphase bridges or nondisjunction/aneuploidy in control untreated cells (-) or auxin-treated (+) cells for 16 hours. $\mathrm{N}=$ 3 experiments (black dots) with $n=15-50$ mitotic cells for each condition and stage of mitosis. Error bars represent standard error. Scale bar in $A-C=10 \mu \mathrm{m}$ and $F=20 \mu \mathrm{m}$. 


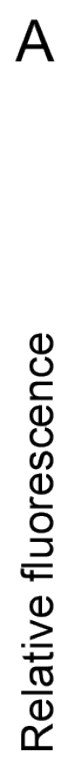
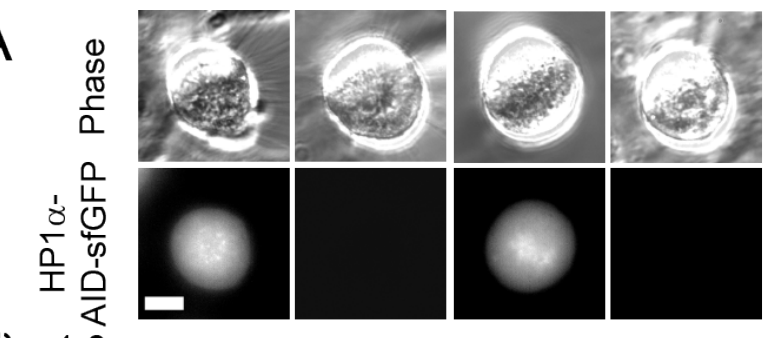

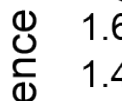

仓

$\begin{array}{rr}0 \\ \stackrel{0}{2} & 0.8 \\ & 0.6\end{array}$

$\$ 0.6$

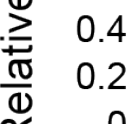

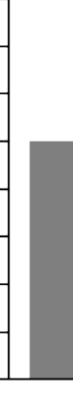

Auxin $(\operatorname{deg} \mathrm{HP} 1 \alpha)$

Methylstat

(his. methylation)

$\mathrm{D}$

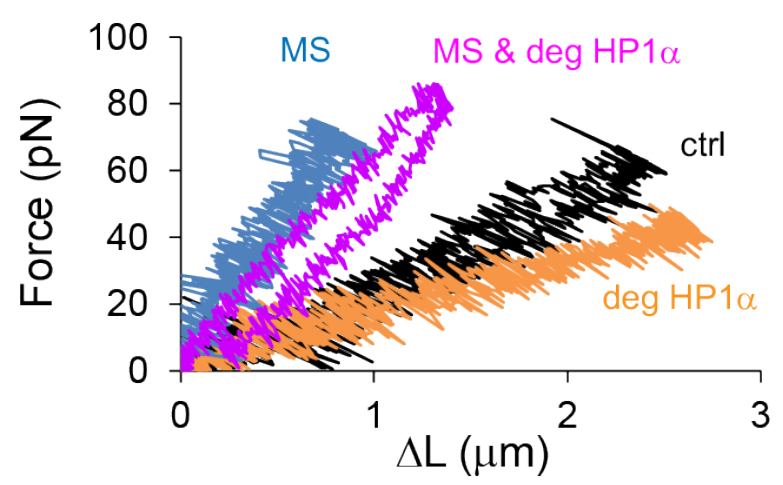

F normal abnormal $G$
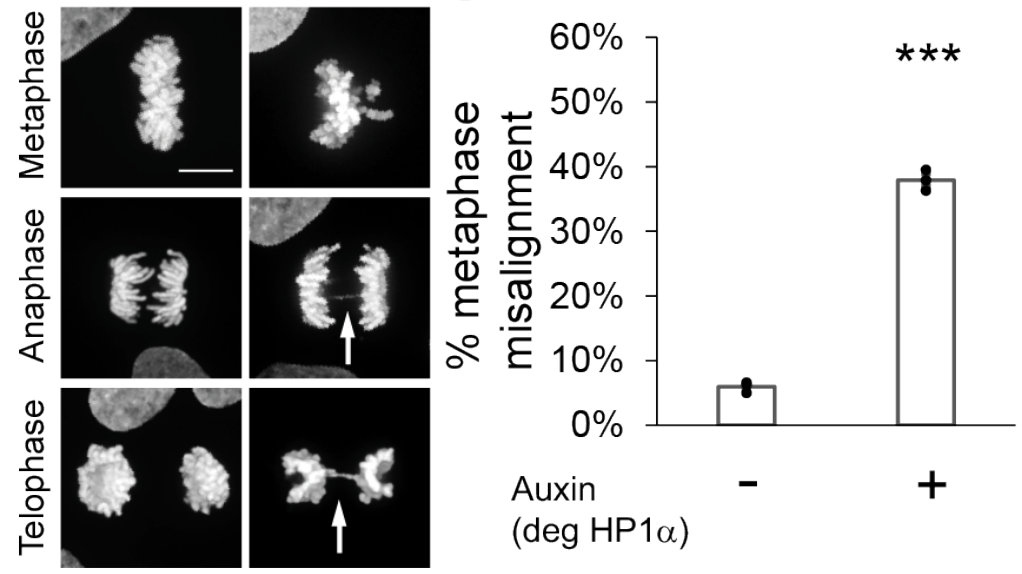

Auxin

(deg HP1 $\alpha$ )
B
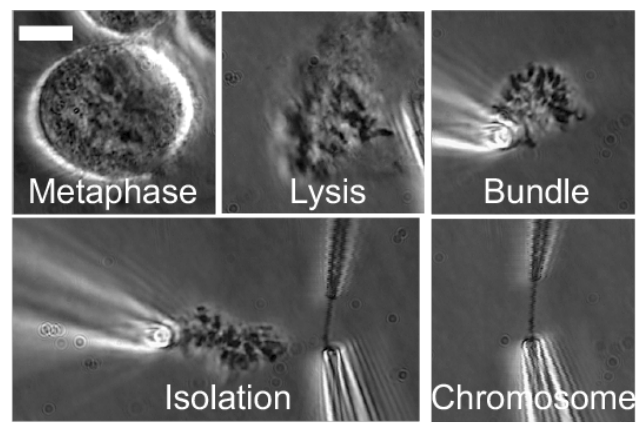

C
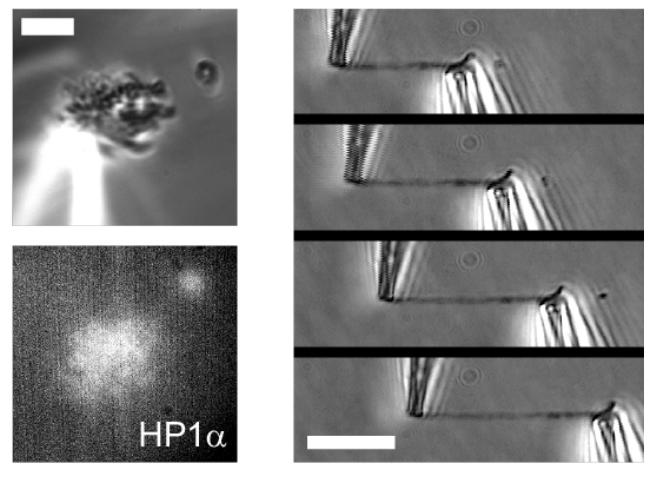

E
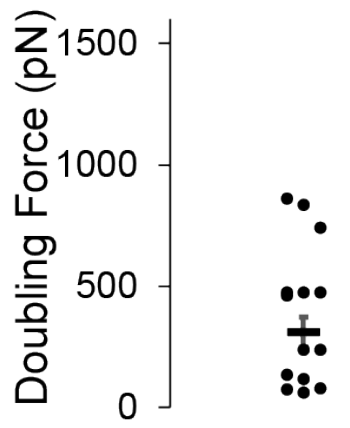

Auxin

(deg HP1 $\alpha$ )

Methylstat

(his. methylation)

$\mathrm{H}$

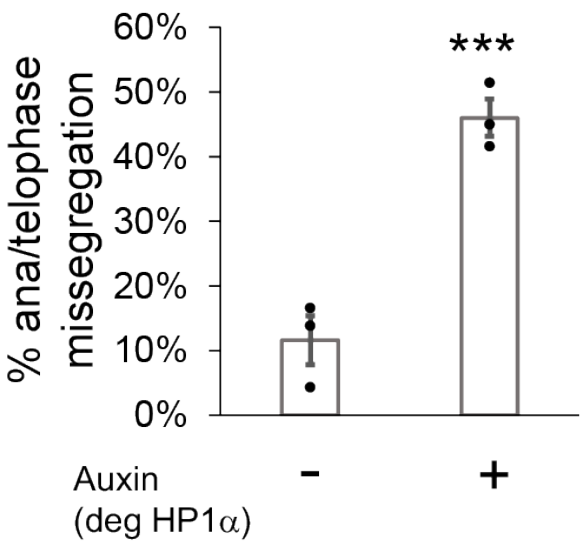




\section{Discussion}

Constitutive heterochromatin comprises an essential nuclear compartment known to perform genome-stabilizing functions through its biochemical and mechanical properties. HP1a is an essential protein component of heterochromatin that orchestrates its structural and functional roles (Kumar and Kono, 2020). To directly characterize these roles, we developed a new tool for rapid and reversible depletion of endogenous HP1 1 p protein through auxin-inducible degradation (Nishimura et al., 2009). Interestingly, rapid and acute depletion of HP1a over four hours does not significantly alter large-scale transcriptional profile or chromatin organization (Figure 1). Nonetheless, acute depletion of HP1 $1 \alpha$ has significant effects on interphase and mitotic chromosome mechanics and morphology (Figures 2-3, 5). Furthermore, HP1a's role is dependent on its ability to dimerize (Figure 3). Together with polymer simulations of interphase nuclear mechanics (Figure 4), these results indicate that HP1 $\alpha$ acts as a dynamic chromatin-chromatin crosslinker to provide mechanical strength to the nucleus, and that this function may persist through mitosis.

\section{HP1a is not essential for transcription repression or heterochromatin compaction on short time scales}

Our data are the first to clearly separate the direct and indirect roles of HP1 $\alpha$ in heterochromatin and its major functions of compaction and transcription. Early studies of HP1 $\alpha$ established its association with compacted regions (beta chromatin) (Bannister et al., 2001), transcriptional silencing in yeast (Fischer et al., 2009; Sadaie et al., 2008), and silencing in Drosophila and mammalian cells at specific sites (Li et al., 2003; Verschure et al., 2005). Recent studies have shown a capacity for HP1 $\alpha$ to suppress transcription in HEK293 cells when overexpressed (Lee et al., 2019) and in MEF cells while recruited to a specific array (Erdel et al., 2020). In contrast, our studies assay global transcription in acute loss of endogenous HP1 $\alpha$ in human cells. We find that acute HP1 $\alpha$ degradation does not result in significant changes in gene transcription and local compaction (Figure 1 and Figure 1 - figure supplement 1), suggesting that its presence is dispensable for maintenance of these heterochromatic features over timescales $<24$ hours.

Chromatin compaction and transcriptional repression also depend on methylation, which promotes HP1 $\alpha$ binding, which in turn may recruit the methyltransferases for further propagation of methylation (Bannister et al., 2001). However, we found that HP1a is not necessary for shortterm maintenance of histone methylation. In particular, after rapid degradation of HP1a by our endogenous auxin-induced degradation construct, there was no significant change in constitutive heterochromatin marker $\mathrm{H} 3 \mathrm{~K} 9 \mathrm{me}^{3}$, commonly associated with transcriptional repression. The lack of widespread changes in transcription agrees with lack of change in $\mathrm{H} 3 \mathrm{~K} 9 \mathrm{me}^{3}$ levels (Figure 2). This is consistent with a previous report that genetic deletion of HP1 $\alpha$ does not alter global $\mathrm{H} 3 \mathrm{~K}_{9} \mathrm{me}^{3}$, but rather, alters specific satellite $\mathrm{H} 3 \mathrm{~K} 9 \mathrm{me}^{3}$ in parts of the genome with repetitive DNA sequences (Bosch-Presegué et al., 2017). Furthermore, our results are supported by the recent 
finding that heterochromatin foci size, compaction, or accessibility are independent of HP1 $\alpha$ binding (Erdel et al., 2020). Together, these results indicate an inability for the transcription machinery to function at heterochromatic loci regardless of whether or not HP1 $\alpha$ is present. Our data also showed that increased histone methylation via methylstat did not result in a global increase in HP1 1 levels.

Altogether, these results are consistent with the existence of a heterochromatin compaction state that is insensitive to the presence or absence of HP1 $\alpha$ (Erdel et al., 2020). Instead, the functional impact of HP1a may appear in processes occurring on longer timescales, for example, DNA replication (Schwaiger et al., 2010), chromosome segregation (Abe et al., 2016), epigenetic imprinting and inheritance (Hathaway et al., 2012; Holla et al., 2020; Nakayama et al., 2000), or post-mitotic reformation of the nucleus (Liu and Pellman, 2020). Nonetheless, as we discuss below, despite its limited impact on global transcription and chromatin organization, HP1a serves an important function as a mechanical stabilizer of the genome and nucleus.

\section{HP1a governs nuclear stiffness with a distinct and separate mechanical contribution from histone methylation}

While acute depletion of HP1a did not alter heterochromatin-specific properties and functions such as histone methylation levels or transcriptional repression, it did significantly contribute to nuclear mechanics. Degradation of HP1 $\alpha$ resulted in a drastic decrease in the short-extension rigidity of the nucleus, reducing the spring constant by $45 \%$ (Figure $2 \mathrm{~B}, \mathrm{D}$ ). Lamin A levels and large-deformation nuclear stiffness, however, were unaffected by HP1 $\alpha$ degradation (Figure 2 B and Figure 2 - figure supplement $1 \mathrm{~A}$ ). These results are consistent with prior experiments showing that chromatin dominates the mechanical response to small deformations, while lamins underlie strain stiffening to large deformations (Stephens et al., 2017). Furthermore, consistent with HP1a's newfound role in chromatin-based mechanics, we find that HP1a degradation results in the loss of nuclear shape stability (Figure $2 \mathrm{E}, \mathrm{G}$ ), similar to the effects of other chromatin perturbations that soften the cell nucleus (Furusawa et al., 2015; Stephens et al., 2019a, 2019b, 2018; Wang et al., 2018). Thus, while acute depletion of HP1a has little apparent effect on genome organization (Figure 1), HP1 $\alpha$ is critical to maintaining the mechanical integrity of chromatin.

It is known that the mechanical contribution of chromatin to the short-extension force response of the nucleus depends on histone modification state (Heo et al., 2016; Hobson et al., 2020; Krause et al., 2019; Liu et al., 2018; Nava et al., 2020; Stephens et al., 2019b, 2018, 2017). We considered the possibility that histone methylation contributes to mechanics through its impact on HP1 $\alpha$ binding to chromatin (Bannister et al., 2001; Erdel et al., 2020; Lachner et al., 2001; Nakayama et al., 2001). However, our experiments show that histone methylation has a distinct contribution to chromatin-based nuclear mechanical response that is largely separate from HP1 $\alpha$ (Figure $2 \mathrm{D}, \mathrm{F}, \mathrm{G}$ ). In particular, nuclear rigidity (and corresponding shape stability) lost by HP1 $\alpha$ 
degradation can be recovered by hypermethylation of histones via methylstat treatment. Furthermore, HP1 $\alpha$ has an additive effect with methylation on nuclear mechanical response: chromatin-based nuclear stiffness decreases after HP1 $\alpha$ degradation with or without treatment with methylstat. Together, these results suggest that HP1 $\alpha$ and histone methylation modulate separable mechanical responses within the cell nucleus. The methylation-based mechanical response may be due to direct interactions between histone marks (Bilokapic et al., 2018; Zhiteneva et al., 2017) or effects of other histone mark readers.

\section{HP1 $\alpha$ contributes to nuclear mechanical response by acting as a chromatin crosslinker}

What is the separate mechanical role of HP1 $\alpha$ in heterochromatin? HP1 $\alpha$ is a homodimer capable of physical bridging chromatin by binding two nucleosomes via methylated histones or chromatin fibers (Canzio et al., 2011; Cheutin et al., 2003; Machida et al., 2018). We found evidence that this capability supports a distinct mechanical function. HP1a's dimerization is essential to its role in maintaining nuclear shape stability (Figure 3), which has been shown here (Figure 2) and previously (Stephens et al., 2019a, 2018) to depend on chromatin-based nuclear stiffness. Thus,

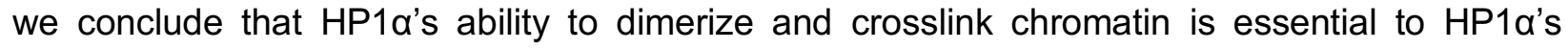
contributions to chromatin-based nuclear stiffness (Figure 6).

This interpretation is supported by coarse-grained polymer simulations of cell nuclear mechanical response. In our model, chromatin is modeled as a crosslinked polymer gel, while the nuclear lamina is modeled as a polymeric shell that is physically linked to the interior chromatin. This model previously recapitulated measurements from nucleus micromanipulation experiments, which observed the two-regime force-extension relationship, its dependence on histone modifications and nuclear lamins, and the changes to the shape of the nucleus when it is stretched (Banigan et al., 2017; Stephens et al., 2017). Here, we showed that the short-extension stiffness, but not the long-extension stiffness, is highly sensitive to the number of chromatin-chromatin crosslinks (Figure $4 \mathrm{C}, \mathrm{D}$ ). Specifically, the short-extension spring constant is small with few crosslinks ( $50 \%$ of the WT simulation), which parallels the result for nuclei with HP1a degraded (Figure 2) and the expected mechanics for nuclei with the HP1 a dimerization mutant (see above). Interestingly, although we model HP1 $\alpha$ as a permanent chromatin-chromatin crosslink, chromatin binding by HP1 $\alpha$ in vivo is transient, with a typical exchange time of $\sim 10$ s (Cheutin et al., 2003; Festenstein et al., 2003; Kilic et al., 2015). Apparently, chromatin-bound HP1a is sufficiently abundant that crosslinks continuously percolate interphase chromatin to provide a robust mechanical response. Simulations with transient crosslinks or experiments with HP1a chromatinbinding mutants (Nielsen et al., 2001) could further investigate this phenomena and its implications for chromatin organization, chromatin-based nuclear mechanics, and nuclear morphology.

More broadly, the finding that HP1 $\alpha$ acts as a chromatin crosslinker is consistent with other experimental data suggesting that chromatin organization and mechanics is supported by 
widespread physical crosslinking. Recent chromosome conformation capture (Hi-C) and micromanipulation experiments show that moderate fragmentation of chromatin does not alter genome organization and mechanics. On this basis of these experiments, it is hypothesized that chromatin may be physically linked as frequently as once per 10-25 kb (Belaghzal et al., 2019). Our data show that HP1 $\alpha$ is one of the likely many possible chromatin crosslinking elements in the genome. There is a growing list of chromatin proteins and nuclear components contributing to maintenance of nuclear morphology, some of which have been identified by a genetic screen for effects on nuclear morphology (Tamashunas et al., 2020) and a variety of other experiments (reviewed in (Stephens et al., 2019a)). Other chromatin crosslinkers to be investigated include chromatin looping proteins and other components implicated by various experiments, such as cohesin, CTCF, mediator, and possibly RNA.

Crosslinking and gelation are intimately coupled to phase separation (Harmon et al., 2017) Therefore, HP1 a may contribute to nuclear mechanics through a phase transition mechanism. In a phase transition model, HP1a dimers crosslinking certain regions of the chromatin polymer would lead to polymer-polymer or sol-gel transitions (Khanna et al., 2019; Tanaka, 2002) that contribute to the elastic modulus of the whole network (Colby and Rubinstein, 2003; Semenov and Rubinstein, 2002; Shivers et al., 2020). Furthermore, HP1 $\alpha$ binding to methylated histones is known to alter the structure of the nucleosome core, which could promote nucleosomenucleosome interactions, and induce polymer-polymer phase separation of the chromatin fiber (Sanulli et al., 2019). Additionally, purified HP1a protein in vitro exhibits liquid-liquid phase separation by itself, with naked DNA, and with nucleosome arrays (Larson et al., 2017; Shakya et al., 2020). The material properties of these in vitro condensates varies depending on the chromatin content (Larson et al., 2017; Shakya et al., 2020). More generally, phase separation in an elastic network such as chromatin can be regulated by the local mechanical properties of the material (Shin et al., 2018; Style et al., 2018). Together, these observations suggest a complex physical picture that is dictated by both HP1 $\alpha$ 's self-interaction and chromatin binding capabilities, in addition to length, concentration, and phase behavior of the chromatin itself (Gibson et al., 2019). The material state and categorization of phase transition of HP1a-rich heterochromatin in vivo have been debated (Erdel et al., 2020; Larson et al., 2017; Strom et al., 2017; Williams et al., 2020), so further work is necessary to completely understand the interplay of these components in determining phase behavior and mechanics of the interphase nucleus. 


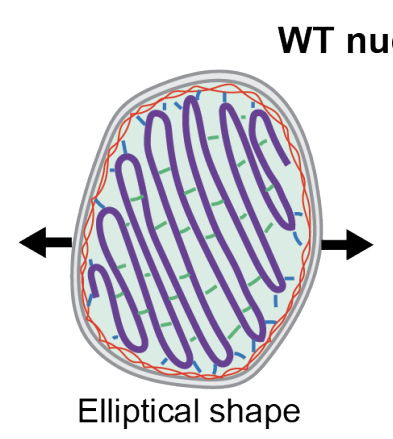

Degraded HP1 $\alpha$

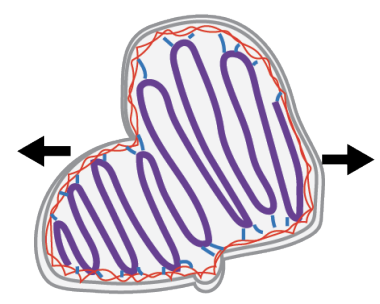

Abnormal shape

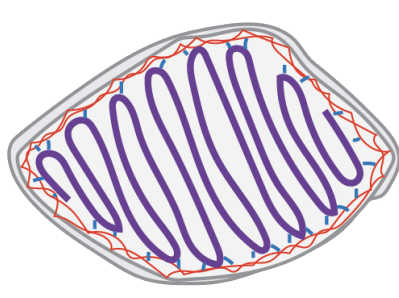

Softer

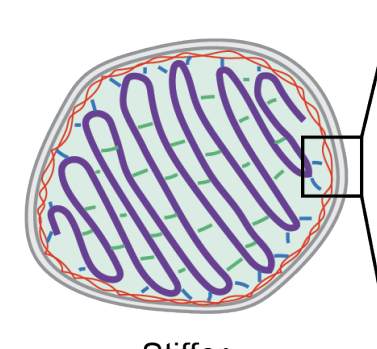

Stiffer
Nuclear Mechanical Components

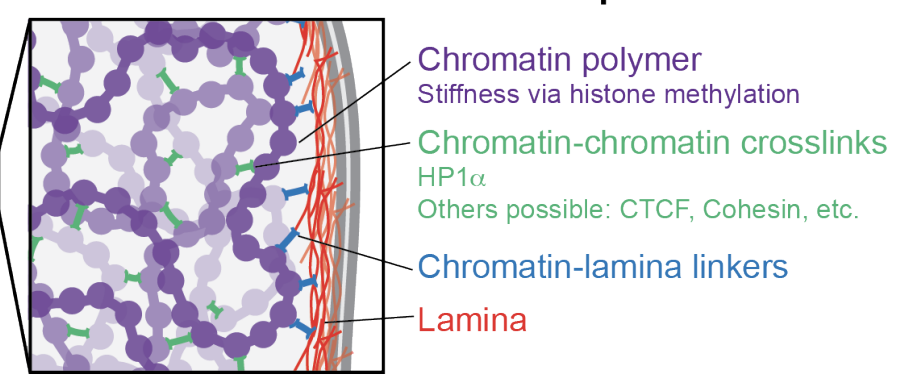

WT Mitotic Chromosomes
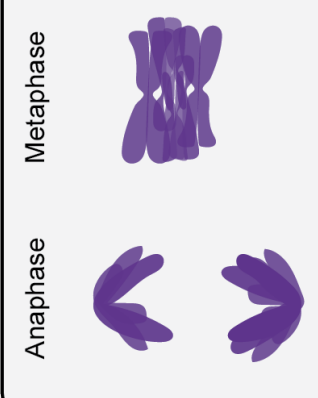

Degraded HP1 $\alpha$

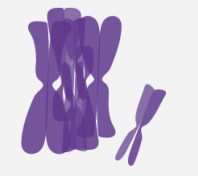

Misalignment

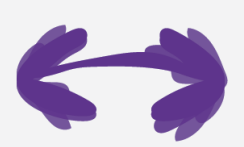

Anaphase bridges

Figure 6. HP1 $\alpha$ is a mechanical element of interphase and mitotic chromosomes.

In Wild-Type (WT) nuclei, HP1 $\alpha$ acts as a chromatin-chromatin crosslinker, resulting in stiffer nuclear mechanics. Other components that contribute to nuclear mechanics include the chromatin polymer (whose mechanical contribution is dictated via histone methylation) the lamina, and chromatin-lamina linkages. Nuclei with HP1a degraded have abnormal shape and softer chromatin-based short-extension mechanical response. Degradation of HP1 1 also leads to softer mitotic chromosomes and mitotic defects, including chromosome misalignment and anaphase bridges.

\section{HP1a is a mechanical element of mitotic chromosomes and is essential for proper mitosis}

Mechanical components of Interphase chromatin may remain in order to maintain the mechanical strength of chromosomes in mitosis. Recent work has shown that heterochromatin-based histone modifications/methylation also control the mechanical strength of chromosomes, while euchromatin-based histone acetylation does not (Biggs et al., 2019). This paper hypothesized that increased histone methylation could be aided by "histone readers" heterochromatin associated proteins, specifically HP1 $1 \alpha$. Our data reveal that, similar to HP1 $\alpha$ in interphase nuclei, HP1 $\alpha$ during mitosis is a significant mechanical component of the mitotic chromosome (Figure 5). Similar to the case in interphase chromosomes, the contributions of histone methylation and HP1 $\alpha$ to the stiffness of mitotic chromosomes are distinct. HP1 $1 \alpha$ degradation leads to more extensible mitotic chromosomes, but much of the stiffness can be recovered by hypermethylation via methylstat treatment. The fact that HP1a still provides mechanical stiffness in mitotic chromosomes, a chromatin only system without lamins, further supports that HP1a mechanical functions as a chromatin crosslinker. Previous work has proposed that mitotic chromosomes are 
dense polymer gels, based on their elastic response, which relies on the continuity of the DNA backbone (Poirier and Marko, 2002) topology (Kawamura et al., 2010), and the chromatin crossbridging condensin protein complex (Sun et al., 2018). Our experiments implicating HP1 1 as a crosslinking element (in interphase) and measuring the mechanical contributions of HP1 $\alpha$ in mitotic chromosomes provide further support for this picture. Methylation could serve as an additional compaction agent by providing further crosslinking, stiffening the chromatin fiber itself, or generating poor solvent conditions that further compact mitotic chromosomes (Batty and Gerlich, 2019; Gibcus et al., 2018; Maeshima et al., 2018). Together, these components generate the rigidity necessary for robust mitotic chromosomes.

Loss of HP1a results in dysfunction, marked by improper chromosome alignment and segregation. Previous reports had noted that loss of HP1 $\alpha$ and HP1 1 , specifically at the centromere, causes mitotic alignment errors (Yi et al., 2018), genetic deletion of HP1 $\alpha$ increases monotelic or syntelic attachments (Bosch-Presegué et al., 2017), and mitosis is dependent on HP1 $\alpha$ phosphorylation (Chakraborty et al., 2014). Our findings with acute degradation of HP1 $\alpha$ reveal a three-fold increase in both misalignment and missegregation, which were mostly observed anaphase bridges. Our results are in agreement with HP1 $\alpha$ interacting with LRIF at the centromere, which when perturbed results in similar misalignment and missegregation (Akram et al., 2018). However, further work is required to determine if chromosome misalignment is due to a biochemical pathway or mechanical pathway at the centromere and if whole chromosome mechanics controlled by HP1a influence proper segregation.

\section{Conclusion}

We have established that HP1 $1 \alpha$ has consistent mechanical and functional implications for chromosomes throughout the cell cycle. While acute degradation of HP1 $\alpha$ has little effect on the global transcriptional profile, loss of HP1a strongly impairs interphase and mitotic chromosome mechanics. This leads to deleterious and potentially catastrophic effects, such as abnormal nuclear morphology and chromosome segregation defects. When present, HP1 $\alpha$ is a crosslinking element, and it mechanically stabilizes interphase and mitotic chromosomes, suppressing nuclear deformations and mitotic defects. It remains unclear whether HP1 1 's phase separation capability is important to this biophysical function. More broadly, our experiments demonstrate that mechanical softening of the nucleus due to acute loss of HP1a's chromatin crosslinking ability, rather than transcriptional changes, could underlie defects in fundamental nuclear functions such as nuclear compartmentalization, DNA damage prevention and response, and migration, all of which have been shown to depend on nuclear mechanics (Stephens, 2020; Xie et al., 2020). These mechanical changes could also have broad implications for human diseases, such as breast cancer, where increased invasiveness (migration ability) has been correlated with decreased HP1a levels (Vad-Nielsen and Nielsen, 2015). Overall, we have revealed a direct structural role for HP1 $\alpha$ in whole-nucleus and mitotic chromosome mechanics that furthers our understanding of chromatin-based nuclear stiffness and has important cellular functional consequences. 


\section{Materials and Methods}

Cloning and characterization of HP1 $\alpha$-AID-sfGFP degron clone. U2OS (ATCC) were cultured in DMEM/FBS and co-transfected with two plasmids, human 3' HP1a-AID- sfGFP 2A PuroR (Addgene 127906) and a guide RNA/Cas9 plasmid pX330 human 3' HP1a gRNA (Addgene 127907) with Lipofectamine 2000 according to manufacturer's instructions. The guide RNA sequence, 5'- acagcaaagagctaaaggag -3', flanked the stop site of the CBX5 gene and was destroyed upon successful in-frame insertion of the AID-GFP 2A PuroR cassette. Modified cells were selected with $10 \mu \mathrm{g} / \mathrm{ml}$ puromycin and single-cell sorted into 96 well plates with a BD FACS Aria III gated with FACSDiva software to sort only the top $10 \%$ brightest GFP-expressing cells. Expression of HP1a-AID-sfGFP was monitored by fluorescence microscopy as clones were expanded and subjected to quality control (QC; quality control, consisting of immunoblotting, PCR and live cell microscopy, see supplementary materials). A homozygous clone that passed all QC (U2OS HP1 $\alpha$ 4) was co-transfected with the transposon vector pEF1a-OsTIR-IRES-NEO-pAT2BH (Addgene 127910) and SB100X in pCAG globin pA (Addgene 127909). 48 hours posttransfection, cells were selected with $400 \mathrm{ug} / \mathrm{ml}$ G418 for 10 days (media with fresh $\mathrm{G} 418$ replaced every 2-3 days) and then allowed to recover in DMEM/FBS for one week. GFP positive cells were again single cell sorted, expanded and subjected to QC. Degradation of HP1a-AID-sfGFP by OsTIR1 was evaluated by flow cytometry, immunoblotting and live cell microscopy after treatment with $1 \mathrm{mM}$ auxin (NaIAA, Sigma \#I5148) for 4-16 hours. A clone (U2OS HP1 $\alpha$ 4-61) that by all QC measures demonstrated no detectable HP1a-AID-sfGFP after auxin treatment was chosen and expanded.

Validation by PCR. Genomic DNA was extracted using the PureLink ${ }^{\text {TM }}$ Genomic DNA Mini Kit (catalog number K182001) and PCR was performed with oligos that flanked the insertion site, yielding 2 PCR products for heterozygous HP1 $\alpha$ clones or a single larger PCR product for HP1 $\alpha$ clones homozygous for the AID-GFP-Puro insertion.

Cell line validation microscopy. Live cells were plated into 4 chambered glass bottomed dishes (Greiner Bio One, \#627975) and mounted in a temperature and $\mathrm{CO}_{2}$ controlled chamber (Okolab) for viewing using a Nikon Eclipse Ti inverted microscope with a 100X, 1.45NA phase objective and Spectra X (Lumencor) LED excitation at DAPI (395/25) and GFP (470/24) wavelengths (used at $5 \%$ power). Cells grown on glass coverslips, fixed in $4 \%$ paraformaldehyde (Polysciences, \#18814) and mounted in Prolong Diamond to preserve GFP signal were also prepared. Images were captured using an Orca Flash 4 sCMOS camera and analyzed, cropped and contrast adjusted for display using either Elements or Imaris software.

Immunoblotting and immunostaining. Cell pellets from each clone were resuspended and incubated in RIPA buffer (Thermo Scientific \# 89901) containing 2x Protease inhibitor (Thermo Scientific \# A32955) for $1 \mathrm{hr}$ on ice, and then incubated for $10 \mathrm{~min}$ at RT with $25 \mathrm{U}$ benzonase nuclease (Millipore Sigma 70746-10KUN)/50 $\mu \mathrm{L}$ sample. After BCA protein quantification (Pierce), samples were subjected to reducing SDS-PAGE and LI-COR Western blot analysis. Anti-HP1 alpha primary antibody (Abcam \#ab109028) was used at 1:250 and IRDye 680CW secondary (LiCOR \#925-6807) was diluted 1:15000. Blots were scanned on an Odyssey $\mathrm{CL}_{\mathrm{x}}$. Immunostaining was carried out as previously described (Politz et al., 2002) using Abcam \#ab109028 primary antibody at 1:250, and secondary antibody (Jackson labs 711-165-152) at 1:200, and coverslips were mounted in Prolong Gold. Images were captured as described above. 
RNA-seq. RNA was isolated using the Qiagen RNeasy kit according to manufacturer's instructions. Cells were homogenized with a QIAshredder (Qiagen \#79654) with $\beta$ mercaptoethanol in the RTL buffer. DNA was digested with RNase-Free DNase (Qiagen \#79254) and purified with RNeasy MinElute Cleanup Kit (Qiagen \#74204). Purified RNA was quantitated with a Nanodrop spectrophotometer and quality was confirmed on a bioanalyzer with a TapeStation R6K assay. A sequencing library from RNA with a RIN > 9.5 was prepared using the TruSeq stranded mRNA Library Prep and sequencing was performed using an Illumina HiSeq 2500 workstation. There were over 16,000 genes with one transcript per million reads for control compared to auxin four hours as well as control compacted to auxin sixteen hours, The RNA-Seq reads were mapped with STAR and then quantified by RSEM, and the differential gene expression analysis was performed using DESeq2.

HP1 $\alpha$ rescue constructs. Full length HP1 $\alpha$ was amplified by PCR (Addgene 17652), and cloned using InFusion kit into a pHR lentiviral vector under an SFFV promoter and tagged C-terminally with mCherry and sspB. A mutation was introduced to disrupt dimerization at amino acid 165 in the chromoshadow domain, changing the codon ATA (coding for Isoleucine, I) to GAG (coding for Glutamic acid, E) to result in HP1 $\alpha^{1165 E}$. This mutation has been previously characterized to disrupt homodimerization of HP1 $\alpha$ (Brasher et al., 2000).

Lentiviral expression of HP1a rescue constructs. LentiX cells were transfected with transfer plasmids pCMV-dR8.91 and pMD2.G, as well as expression construct of interest in a 9:8:1 mass ratio into HEK293T cells using FuGENE HD Transfection Reagent (Promega) per manufacturer's protocol. After 48 hours, media containing viral particles was collected and filtered using 0.45 micron filter (Pall Life Sciences), and either used immediately or stored at $-80{ }^{\circ} \mathrm{C}$. HP1a-AIDsfGFP cells were plated at $15-20 \%$ confluency on glass-bottom 96 -well plates (Cellvis) and infected with 10-50 $\mu \mathrm{L}$ of virus-containing media. After 24 hours, viral media was removed and replaced with fresh DMEM, and cells were fixed or imaged at 3-7 days post-infection.

Immunostain, microscopy, and morphological analysis of nuclear shape in fixed cells. HP1 $\alpha$-AID-sfGFP cells expressing mCherry-tagged HP1 $\alpha^{\text {WT }}$ or HP1 $\alpha^{1165 E}$ were treated with control media (no auxin) or $1 \mathrm{mM}$ auxin (NalAA, Sigma \#I5148) for 16 hours before being fixed in $4 \%$ paraformaldehyde for 10 minutes, washed three times in PBS, permeabilized with $1 \%$ triton X100 in PBS for 1 hour at room temperature with rocking, blocked with 5\% FBS in $0.25 \%$ PBST for 1 hour at room temperature with rocking, and incubated with anti-Lamin A/C antibody 1:1000 (Active Motif, 39287) in block overnight. Samples were washed again three times with PBS and incubated with Goat anti-mouse IgG secondary antibody conjugated to Alexa fluor 647 (Thermo Fisher, A-21236) for $>2$ hours, washed again and incubated with Hoechst 1:2000 in PBS for 30 minutes. Images of fixed and stained cells were obtained with a spinning-disk confocal microscope (Yokogawa CSU-X1) with 100X oil immersion Apo TIRF objective (NA 1.49) and Andor DU-897 EMCCD camera on a Nikon Eclipse Ti body. Live samples were maintained at $37^{\circ} \mathrm{C}$ and $5 \% \mathrm{CO}_{2}$ by a 96-well plate incubation chamber (Okolab). 405, 488, 561 and 647 lasers were used for imaging Hoechst, sfGFP, mCherry or Alexa 568, and Alexa 647, respectively. Laser power and digital gain were consistent for imaging all samples across an experiment, allowing for quantitative comparison of fluorescent intensities. Morphological analysis was performed in FIJI using a plugin that measures curvature; Kappa, which was created originally by Kevan Lu and is now maintained by Hadrien Mary. Briefly, one z-slice of the Lamin A/C immunostain channel at the center of the height of the nucleus was loaded into the Kappa plugin, traced, and a closed curve was fit to the signal. Curvature along the nuclear envelope trace was calculated as the 
inverse radius of curvature with the plugin and an average value of curvature per nucleus was recorded.

Microscopy and morphological analysis of nuclear shape in living cells. Control media (no auxin) or $1 \mathrm{mM}$ auxin (NaIAA, Sigma \#15148) was added to HP1a-AID-sfGFP cells expressing an miRFP-tagged histone $\mathrm{H} 2 \mathrm{~B}$ plated in 96-well glass bottom plates (Cellvis). $25 \mathrm{X}-\mathrm{Y}$ points were chosen in each of the control and experimental wells, and a z-stack ranging 8 microns was collected at each point every 30 minutes for 12 hours (with Auxin added to experimental wells at time 0 hours). Morphological analysis was again performed with the FIJI plugin Kappa, this time using the histone signal to delineate the edge of the nucleus.

Cell protocol for single nucleus and mitotic chromosome isolation. Micromanipulation experiments used U2OS parent or HP1 1 -AID-sfGFP cells maintained in DMEM (Corning) with $10 \%$ fetal bovine serum (FBS) (HyClone) and 1\% 100x penicillin streptomycin (Corning). The cells were plated and allowed to recover 1-3 days before nucleus or chromosome isolation. $1 \mathrm{mM}$ auxin (NaIAA, Sigma \#I5148) was added 4 to 6 hours before nucleus and chromosome isolation in "+ auxin" and "+ auxin, + methylstat" experiments, and $1 \mathrm{mM}$ methylstat was added 30-38 $\mathrm{h}$ before "+ methylstat" and "+ auxin, + methylstat" experiments. All experiments were performed without synchronization.

Single nucleus and mitotic chromosome isolation. Single nucleus (Stephens et al., 2017) and chromosome isolation (Biggs et al., 2019) experiments were performed on an inverted microscope (IX-70; Olympus) with a 60x 1.42 NA oil immersion objective with a 1.5x magnification pullout. Nuclei and Chromosomes were isolated at room temperature and atmospheric $\mathrm{CO}_{2}$ levels in DMEM $10 \%$ FBS $1 \%$ pen strep media in 3 hours or less to ensure minimal damage to the cells and chromosomes. Before isolation all cells were imaged for the absence or presence of HP1 $\alpha$ with or without auxin treatment, respectively. For nucleus isolation, cells were treated with $1 \mu \mathrm{g} /$ $\mathrm{mL}$ latrunculin A (Enzo Life Sciences) for 45 minutes before isolation to depolymerize the actin cytoskeleton. Interphase cells were lysed with $0.05 \%$ Triton-X 100 in PBS. After lysis, micromanipulation pipettes filled with PBS were used to capture and position the single isolated nucleus. Isolation aimed for G1 nuclei determined by their size (10 - $15 \mu \mathrm{m}$ along the major axis). For chromosomes, prometaphase mitotic cells were identified by eye and lysed with $0.05 \%$ Triton$X 100$ in PBS. After lysis, the bundle of interconnected chromosomes fell out of the cell and stabilized with a PBS filled pipette by light aspiration. While the bundle was stabilized, one end of a loose chromosome was aspirated into an easily bendable (Kavg=40 pN/ $\mu \mathrm{m}$ ) "force" pipette, moved away from the bundle, where the other end of the chromosome was grabbed by a stiff pipette. The bundle was heavily aspirated into the stabilizing pipette and then removed, leaving an isolated chromosome to be manipulated.

Nucleus mechanics measurements. The isolated nucleus is suspended between a stiff pull pipette and a pre-calibrated force pipette for defined size (2.8 to $3.3 \mu \mathrm{m}$ diameter) and bending constant (1.5-2.0 $\mathrm{nN} / \mu \mathrm{m})$. The pull pipette provides either $3 \mu \mathrm{m}$ extension (short regime only) or $6 \mu \mathrm{m}$ extension of the nucleus (long regime) at a rate of $0.05 \mu \mathrm{m} / \mathrm{sec}$. Bending of the force pipette relative to extension of the nucleus provides a measure of force. Data is transferred to Excel where the slope of the force-extension provides a nuclear spring constant for chromatin (short extension 0-3 $\mu \mathrm{m}$ extension) and a lamin-based strain-stiffening nuclear spring constant (long regime slope minus short regime slope). 
Mitotic chromosome mechanics measurements. Once a mitotic chromosome was isolated, the stiff pipette was moved $6.0 \mu \mathrm{m}$ at a rate of $0.20 \mu \mathrm{m} / \mathrm{sec}$ with step sizes of $0.04 \mu \mathrm{m} / \mathrm{step}$ using a Labview program, while the force pipette (Fp) and stiff pipette (Sp) were visually tracked. A linear regression of the deflection vs stretch $(\mathrm{Fp} /(\mathrm{Sp}-\mathrm{Fp}))$ slope was calculated, multiplied by the force pipette spring constant (calibrated after the experiment) to give the spring constant of the chromosome, and multiplied by the initial length of the chromosome, to give the doubling force of the chromosome in a custom Python script.

Mitotic chromosome fluorescence. Cells were imaged on an inverted microscope (IX-70; Olympus) with a $60 \times 1.42$ NA oil immersion objective with a $1.5 \times$ magnification pullout. in the GFP channel once a mitotic cell was identified and the final isolated chromosome was imaged in the GFP channel for each experiment to determine if they contained HP1a. Periodically, the chromosome bundle was also imaged in the GFP channel.

Nuclear Morphology measurements. Nuclei were selected via intensity threshold in Hoechst channel and made into an object or ROI and reported for nuclear solidity, which measures convex area of concave hull around the nucleus. The threshold of 0.97 solidity was used to determine normal versus abnormally shaped nuclei. Alternatively, nuclei stained with lamin $A / C$ were loaded into FIJI program Kappa where the outline trace provided by lamin A/C allowed for measurement of nucleus curvature, which is the inverse of the radius of curvature. 
Brownian dynamics simulations. Brownian dynamics simulations of a polymeric shell linked to an interior crosslinked polymer were performed as described previously (Banigan et al., 2017; Stephens et al., 2017). 1000 shell subunits with diameter $a_{s}=0.71 \mu \mathrm{m}$ are randomly placed on a sphere of radius $R_{\mathrm{i}}=10 \mu \mathrm{m}$, which is shrunk to $R_{0}=5 \mu \mathrm{m}$ during the simulation initialization (Banigan Biophys $\mathrm{J} 2017$ ). Each shell subunit is connected by springs to $4 \leq z \leq 8$ nearest neighbor shell subunits $(<z>\approx 4.5)$. A linear polymeric chain of 552 subunits with diameter $a_{p}=$ $0.57 \mu \mathrm{m}$, connected by springs, is initialized in a random globular conformation within the shell. The polymer is randomly crosslinked with $N_{C}$ crosslinks, where $N_{C}=55(20 \%$ of all polymer subunits are crosslinked) unless noted. $N_{\mathrm{L}}$ polymer subunits near the surface of the sphere are linked by springs to the nearest shell subunit; $N_{\mathrm{L}}=40$ (i.e., $7.2 \%$ of all polymer subunits or $22 \%$ of all peripheral subunits, defined by contact with the shell subunits in the initial configuration, are linked to the shell) unless noted. Tensile force is exerted across the nucleus by exerting force $F$ along the $x$-axis on a single shell subunit at each of the two poles.

Spring potentials governing interactions between subunits have the form $U_{\mathrm{sp}}=\left(k_{\mathrm{sp}} / 2\right)\left(r_{\mathrm{ij}}-r_{\mathrm{ij}, 0}\right)^{2}$ for $r_{\mathrm{ij}}>r_{\mathrm{ij}, 0}$, where $r_{\mathrm{ij}}$ is the distance between subunits $i$ and $j, r_{\mathrm{ij}, 0}$ is the sum of the two subunit radii, and $k_{\mathrm{sp}}$ is the spring constant, which depends on the type of potential. $k_{\mathrm{s}}=0.8 \mathrm{nN} / \mu \mathrm{m}$ for shellshell springs, $k_{\mathrm{p}}=1.6 \mathrm{nN} / \mu \mathrm{m}$ unless noted for "polymer springs" connecting subunits along the polymer backbone, $k_{\mathrm{C}}=k_{\mathrm{p}}$ unless noted for "crosslink springs" connecting polymer subunits, and $k_{\mathrm{L}}=k_{\mathrm{p}}$ for springs linking the polymer to the shell. All subunits repel each other via soft-core excluded volume interactions, modeled as $U_{\mathrm{ex}}=\left(k_{\mathrm{ex}, \mathrm{j}} / 2\right)\left(r_{\mathrm{ij}}-r_{\mathrm{ij}, 0}\right)^{2}$ for $r_{\mathrm{ij}}<r_{\mathrm{ij}, 0}$, where $k_{\mathrm{ij}}$ is the repulsive spring constant; $k_{\mathrm{ex}, \mathrm{ij}}=k_{\mathrm{s}}$ if $i$ and $j$ are both shell subunits, $k_{\mathrm{ex}, \mathrm{ij}}=k_{\mathrm{p}}$ if $i$ and $j$ are both polymer subunits, and $k_{\mathrm{ex}, \mathrm{ij}}=2 k_{\mathrm{s}} k_{\mathrm{p}} /\left(k_{\mathrm{s}}+k_{\mathrm{p}}\right)$ if one is a shell subunit and the other is a polymer subunit.

All subunits are subject to uncorrelated thermal noise ( $T=300 \mathrm{~K}$ ). The system obeys the overdamped Langevin equation, which is solved by an Euler algorithm (Allen and Tildesley, 1987) with timestep $d t=0.0005$. 


\section{Acknowledgements}

This work was supported by the NIH Center for 3D Structure and Physics of the Genome of the 4DN Consortium (U54DK107980) and the NIH Physical Sciences-Oncology Center (U54CA193419). CPB and ARS were supported by the Howard Hughes Medical Institute, and grants from the NIH 4D Nucleome Program (U01 DA040601); ARS is supported by the LSRF Fellowship from Mark Foundation For Cancer Research. EJB was supported by the NIH Center for 3D Structure and Physics of the Genome of the 4DN Consortium (U54DK107980), the NIH Physical Sciences-Oncology Center (U54CA193419), and NIH grant GM114190. XW and FY are supported by 1R35GM124820, R01HG009906, U01CA200060 and R24DK106766. KC and ADS are supported by the Pathway to Independence Award (R00GM123195) and 4D Nucleome center grant (1UM1HG011536). 


\section{Bibliography}

Abe Y, Sako K, Takagaki K, Hirayama Y, Uchida KSK, Herman JA, DeLuca JG, Hirota T. 2016. HP1-Assisted Aurora B Kinase Activity Prevents Chromosome Segregation Errors. Dev Cell 36:487-497.

Akram S, Yang F, Li J, Adams G, Liu Y, Zhuang X, Chu L, Liu X, Emmett N, Thompson W, Mullen M, Muthusamy S, Wang W, Mo F, Liu X. 2018. LRIF1 interacts with HP1a to coordinate accurate chromosome segregation during mitosis. Journal of Molecular Cell Biology. doi:10.1093/jmcb/mjy040

Allen MP, Tildesley DJ. 1987. Computer simulation of liquids. Oxford: Clarendon Press.

Azzaz AM, Vitalini MW, Thomas AS, Price JP, Blacketer MJ, Cryderman DE, Zirbel LN, Woodcock CL, Elcock AH, Wallrath LL, Shogren-Knaak MA. 2014. Human heterochromatin protein 1 a promotes nucleosome associations that drive chromatin condensation. $J$ Biol Chem 289:6850-6861.

Banigan EJ, Stephens AD, Marko JF. 2017. Mechanics and Buckling of Biopolymeric Shells and Cell Nuclei. Biophys J 113:1654-1663.

Bannister AJ, Zegerman P, Partridge JF, Miska EA, Thomas JO, Allshire RC, Kouzarides T. 2001. Selective recognition of methylated lysine 9 on histone H3 by the HP1 chromo domain. Nature 410:120-124.

Batty P, Gerlich DW. 2019. Mitotic Chromosome Mechanics: How Cells Segregate Their Genome. Trends Cell Biol 29:717-726.

Belaghzal H, Borrman T, Stephens AD, Lafontaine DL. 2019. Compartment-dependent chromatin interaction dynamics revealed by liquid chromatin Hi-C. bioRxiv.

Biggs R, Liu PZ, Stephens AD, Marko JF. 2019. Effects of altering histone posttranslational modifications on mitotic chromosome structure and mechanics. Mol Biol Cell 30:820-827.

Bilokapic S, Strauss M, Halic M. 2018. Cryo-EM of nucleosome core particle interactions in trans. Sci Rep 8:7046.

Bosch-Presegué L, Raurell-Vila H, Thackray JK, González J, Casal C, Kane-Goldsmith N, Vizoso M, Brown JP, Gómez A, Ausió J, Zimmermann T, Esteller M, Schotta G, Singh PB, Serrano L, Vaquero A. 2017. Mammalian HP1 Isoforms Have Specific Roles in Heterochromatin Structure and Organization. Cell Rep 21:2048-2057.

Brasher SV, Smith BO, Fogh RH, Nietlispach D, Thiru A, Nielsen PR, Broadhurst RW, Ball LJ, Murzina NV, Laue ED. 2000. The structure of mouse HP1 suggests a unique mode of single peptide recognition by the shadow chromo domain dimer. EMBO J 19:1587-1597.

Canzio D, Chang EY, Shankar S, Kuchenbecker KM, Simon MD, Madhani HD, Narlikar GJ, AlSady B. 2011. Chromodomain-mediated oligomerization of HP1 suggests a nucleosomebridging mechanism for heterochromatin assembly. Mol Cell 41:67-81.

Chakraborty A, Prasanth KV, Prasanth SG. 2014. Dynamic phosphorylation of HP1a regulates mitotic progression in human cells. Nat Commun 5:3445.

Cheutin T, McNairn AJ, Jenuwein T, Gilbert DM, Singh PB, Misteli T. 2003. Maintenance of stable heterochromatin domains by dynamic HP1 binding. Science 299:721-725.

Chu L, Huo Y, Liu X, Yao P, Thomas K, Jiang H, Zhu T, Zhang G, Chaudhry M, Adams G, Thompson W, Dou Z, Jin C, He P, Yao X. 2014. The spatiotemporal dynamics of chromatin protein $\mathrm{HP} 1 \alpha$ is essential for accurate chromosome segregation during cell division. $J$ Biol Chem 289:26249-26262.

Colby RH, Gillmor JR, Rubinstein M. 1993. Dynamics of near-critical polymer gels. Phys Rev $E$ Stat Phys Plasmas Fluids Relat Interdiscip Topics 48:3712-3716.

Colby RH, Rubinstein M. 2003. Polymer physics. New-York: Oxford University.

Doudna JA, Charpentier E. 2014. Genome editing. The new frontier of genome engineering with CRISPR-Cas9. Science 346:1258096. 
Erdel F, Rademacher A, Vlijm R, Tünnermann J, Frank L, Weinmann R, Schweigert E, Yserentant K, Hummert J, Bauer C, Schumacher S, Al Alwash A, Normand C, Herten D-P, Engelhardt J, Rippe K. 2020. Mouse Heterochromatin Adopts Digital Compaction States without Showing Hallmarks of HP1-Driven Liquid-Liquid Phase Separation. Mol Cell 78:236-249.e7.

Festenstein R, Pagakis SN, Hiragami K, Lyon D, Verreault A, Sekkali B, Kioussis D. 2003. Modulation of heterochromatin protein 1 dynamics in primary Mammalian cells. Science 299:719-721.

Fischer T, Cui B, Dhakshnamoorthy J, Zhou M, Rubin C, Zofall M, Veenstra TD, Grewal SIS. 2009. Diverse roles of HP1 proteins in heterochromatin assembly and functions in fission yeast. Proc Natl Acad Sci U S A 106:8998-9003.

Fischle W, Tseng BS, Dormann HL, Ueberheide BM, Garcia BA, Shabanowitz J, Hunt DF, Funabiki H, David Allis C. 2005. Regulation of HP1-chromatin binding by histone H3 methylation and phosphorylation. Nature 438:1116-1122.

Frescas D, Guardavaccaro D, Kuchay SM, Kato H, Poleshko A, Basrur V, Elenitoba-Johnson KS, Katz RA, Pagano M. 2008. KDM2A represses transcription of centromeric satellite repeats and maintains the heterochromatic state. Cell Cycle 7:3539-3547.

Furusawa T, Rochman M, Taher L, Dimitriadis EK, Nagashima K, Anderson S, Bustin M. 2015. Chromatin decompaction by the nucleosomal binding protein HMGN5 impairs nuclear sturdiness. Nat Commun 6:6138.

Gibcus JH, Samejima K, Goloborodko A, Samejima I, Naumova N, Nuebler J, Kanemaki MT, Xie L, Paulson JR, Earnshaw WC, Mirny LA, Dekker J. 2018. A pathway for mitotic chromosome formation. Science 359. doi:10.1126/science.aao6135

Gibson BA, Doolittle LK, Schneider MWG, Jensen LE, Gamarra N, Henry L, Gerlich DW, Redding S, Rosen MK. 2019. Organization of Chromatin by Intrinsic and Regulated Phase Separation. Cell 179:470-484.e21.

Hahn M, Dambacher S, Dulev S, Kuznetsova AY, Eck S, Wörz S, Sadic D, Schulte M, Mallm J$P$, Maiser A, Debs P, von Melchner H, Leonhardt H, Schermelleh L, Rohr K, Rippe K, Storchova Z, Schotta G. 2013. Suv4-20h2 mediates chromatin compaction and is important for cohesin recruitment to heterochromatin. Genes Dev 27:859-872.

Harmon TS, Holehouse AS, Rosen MK, Pappu RV. 2017. Intrinsically disordered linkers determine the interplay between phase separation and gelation in multivalent proteins. Elife 6. doi:10.7554/eLife.30294

Hathaway NA, Bell O, Hodges C, Miller EL, Neel DS, Crabtree GR. 2012. Dynamics and memory of heterochromatin in living cells. Cell 149:1447-1460.

Heo S-J, Driscoll TP, Thorpe SD, Nerurkar NL, Baker BM, Yang MT, Chen CS, Lee DA, Mauck RL. 2016. Differentiation alters stem cell nuclear architecture, mechanics, and mechanosensitivity. Elife 5. doi:10.7554/eLife.18207

Hirota T, Lipp JJ, Toh B-H, Peters J-M. 2005. Histone H3 serine 10 phosphorylation by Aurora B causes HP1 dissociation from heterochromatin. Nature 438:1176-1180.

Hobson CM, Kern M, O’Brien ET 3rd, Stephens AD, Falvo MR, Superfine R. 2020. Correlating nuclear morphology and external force with combined atomic force microscopy and light sheet imaging separates roles of chromatin and lamin $A / C$ in nuclear mechanics. Mol Biol Cell 31:1788-1801.

Hobson CM, Stephens AD. 2020. Modeling of Cell Nuclear Mechanics: Classes, Components, and Applications. Cells 9. doi:10.3390/cells9071623

Holla S, Dhakshnamoorthy J, Folco HD, Balachandran V, Xiao H, Sun L-L, Wheeler D, Zofall M, Grewal SIS. 2020. Positioning Heterochromatin at the Nuclear Periphery Suppresses Histone Turnover to Promote Epigenetic Inheritance. Cell 180:150-164.e15.

Jacobs SA, Khorasanizadeh S. 2002. Structure of HP1 chromodomain bound to a lysine 9methylated histone H3 tail. Science 295:2080-2083. 
James TC, Elgin SC. 1986. Identification of a nonhistone chromosomal protein associated with heterochromatin in Drosophila melanogaster and its gene. Mol Cell Bio/ 6:3862-3872.

Kawamura R, Pope LH, Christensen MO, Sun M, Terekhova K, Boege F, Mielke C, Andersen $\mathrm{AH}$, Marko JF. 2010. Mitotic chromosomes are constrained by topoisomerase II-sensitive DNA entanglements. J Cell Biol 188:653-663.

Khanna N, Zhang Y, Lucas JS, Dudko OK, Murre C. 2019. Chromosome dynamics near the solgel phase transition dictate the timing of remote genomic interactions. Nat Commun 10:2771.

Kilic S, Bachmann AL, Bryan LC, Fierz B. 2015. Multivalency governs HP1 $\alpha$ association dynamics with the silent chromatin state. Nat Commun 6:7313.

Krause M, Yang FW, Te Lindert M, Isermann P, Schepens J, Maas RJA, Venkataraman C, Lammerding J, Madzvamuse A, Hendriks W, Te Riet J, Wolf K. 2019. Cell migration through three-dimensional confining pores: speed accelerations by deformation and recoil of the nucleus. Philos Trans R Soc Lond B Biol Sci 374:20180225.

Krouwels IM, Wiesmeijer K, Abraham TE, Molenaar C, Verwoerd NP, Tanke HJ, Dirks RW. 2005. A glue for heterochromatin maintenance: stable SUV39H1 binding to heterochromatin is reinforced by the SET domain. J Cell Biol 170:537-549.

Kumar A, Kono H. 2020. Heterochromatin protein 1 (HP1): interactions with itself and chromatin components. Biophys Rev 12:387-400.

Lachner M, O'Carroll D, Rea S, Mechtler K, Jenuwein T. 2001. Methylation of histone H3 lysine 9 creates a binding site for HP1 proteins. Nature 410:116-120.

Larson AG, Elnatan D, Keenen MM, Trnka MJ, Johnston JB, Burlingame AL, Agard DA, Redding S, Narlikar GJ. 2017. Liquid droplet formation by HP1a suggests a role for phase separation in heterochromatin. Nature 547:236-240.

Lee DH, Ryu HW, Kim GW, Kwon SH. 2019. Comparison of three heterochromatin protein 1 homologs in. J Cell Sci 132. doi:10.1242/jcs.222729

Lionetti MC, Bonfanti S, Fumagalli MR, Budrikis Z, Font-Clos F, Costantini G, Chepizhko O, Zapperi S, La Porta CAM. 2020. Chromatin and Cytoskeletal Tethering Determine Nuclear Morphology in Progerin-Expressing Cells. Biophys J 118:2319-2332.

Liu K, Patteson AE, Banigan EJ, Schwarz JM. 2020. Dynamic nuclear structure emerges from chromatin crosslinks and motors. bioRxiv.

Liu L, Luo Q, Sun J, Ju Y, Morita Y, Song G. 2018. Chromatin organization regulated by EZH2mediated $\mathrm{H} 3 \mathrm{~K} 27 \mathrm{me} 3$ is required for OPN-induced migration of bone marrow-derived mesenchymal stem cells. Int J Biochem Cell Biol 96:29-39.

Liu S, Pellman D. 2020. The coordination of nuclear envelope assembly and chromosome segregation in metazoans. Nucleus 11:35-52.

Li Y, Danzer JR, Alvarez P, Belmont AS, Wallrath LL. 2003. Effects of tethering HP1 to euchromatic regions of the Drosophila genome. Development 130:1817-1824.

Machida S, Takizawa Y, Ishimaru M, Sugita Y, Sekine S, Nakayama J-I, Wolf M, Kurumizaka H. 2018. Structural Basis of Heterochromatin Formation by Human HP1. Mol Cell 69:385397.e8.

Maeshima K, Matsuda T, Shindo Y, Imamura H, Tamura S, Imai R, Kawakami S, Nagashima R, Soga T, Noji H, Oka K, Nagai T. 2018. A Transient Rise in Free Mg2+ lons Released from ATP-Mg Hydrolysis Contributes to Mitotic Chromosome Condensation. Curr Biol 28:444451.e6.

Nakayama J, Klar AJS, Grewal SIS. 2000. A chromodomain protein, Swi6, performs imprinting functions in fission yeast during mitosis and meiosis. Cell.

Nakayama J, Rice JC, Strahl BD, Allis CD, Grewal SI. 2001. Role of histone H3 lysine 9 methylation in epigenetic control of heterochromatin assembly. Science 292:110-113.

Nava MM, Miroshnikova YA, Biggs LC, Whitefield DB, Metge F, Boucas J, Vihinen H, Jokitalo E, Li X, García Arcos JM, Hoffmann B, Merkel R, Niessen CM, Dahl KN, Wickström SA. 2020. 
Heterochromatin-Driven Nuclear Softening Protects the Genome against Mechanical Stress-Induced Damage. Cell 181:800-817.e22.

Nielsen AL, Oulad-Abdelghani M, Ortiz JA, Remboutsika E, Chambon P, Losson R. 2001. Heterochromatin formation in mammalian cells: interaction between histones and HP1 proteins. Mol Cell 7:729-739.

Nielsen PR, Nietlispach D, Mott HR, Callaghan J, Bannister A, Kouzarides T, Murzin AG, Murzina NV, Laue ED. 2002. Structure of the HP1 chromodomain bound to histone H3 methylated at lysine 9. Nature 416:103-107.

Nishimura K, Fukagawa T, Takisawa H, Kakimoto T, Kanemaki M. 2009. An auxin-based degron system for the rapid depletion of proteins in nonplant cells. Nat Methods 6:917-922.

Poirier MG, Marko JF. 2002. Mitotic chromosomes are chromatin networks without a mechanically contiguous protein scaffold. Proc Natl Acad Sci U S A 99:15393-15397.

Poleshko A, Mansfield KM, Burlingame CC, Andrake MD, Shah NR, Katz RA. 2013. The human protein PRR14 tethers heterochromatin to the nuclear lamina during interphase and mitotic exit. Cell Rep 5:292-301.

Polioudaki H, Kourmouli N, Drosou V, Bakou A, Theodoropoulos PA, Singh PB, Giannakouros T, Georgatos SD. 2001. Histones H3/H4 form a tight complex with the inner nuclear membrane protein LBR and heterochromatin protein 1. EMBO Rep 2:920-925.

Politz JC, Lewandowski LB, Pederson T. 2002. Signal recognition particle RNA localization within the nucleolus differs from the classical sites of ribosome synthesis. J Cell Biol 159:411-418.

Ribeiro SA, Gatlin JC, Dong Y, Joglekar A, Cameron L, Hudson DF, Farr CJ, McEwen BF, Salmon ED, Earnshaw WC, Vagnarelli P. 2009. Condensin regulates the stiffness of vertebrate centromeres. Mol Biol Cell 20:2371-2380.

Sadaie M, Kawaguchi R, Ohtani Y, Arisaka F, Tanaka K, Shirahige K, Nakayama J-I. 2008. Balance between distinct HP1 family proteins controls heterochromatin assembly in fission yeast. Mol Cell Biol 28:6973-6988.

Sanulli S, Trnka MJ, Dharmarajan V, Tibble RW, Pascal BD, Burlingame AL, Griffin PR, Gross JD, Narlikar GJ. 2019. HP1 reshapes nucleosome core to promote phase separation of heterochromatin. Nature 575:390-394.

Schindelin J, Arganda-Carreras I, Frise E, Kaynig V, Longair M, Pietzsch T, Preibisch S, Rueden C, Saalfeld S, Schmid B, Tinevez J-Y, White DJ, Hartenstein V, Eliceiri K, Tomancak P, Cardona A. 2012. Fiji: an open-source platform for biological-image analysis. Nat Methods 9:676-682.

Schotta G. 2002. Central role of Drosophila SU(VAR)3-9 in histone H3-K9 methylation and heterochromatic gene silencing. The EMBO Journal. doi:10.1093/emboj/21.5.1121

Schreiner SM, Koo PK, Zhao Y, Mochrie SGJ, King MC. 2015. The tethering of chromatin to the nuclear envelope supports nuclear mechanics. Nat Commun 6:7159.

Schwaiger M, Kohler H, Oakeley EJ, Stadler MB, Schübeler D. 2010. Heterochromatin protein 1 (HP1) modulates replication timing of the Drosophila genome. Genome Res 20:771-780.

Semenov AN, Rubinstein M. 2002. Dynamics of entangled associating polymers with large aggregates. Macromolecules.

Serrano A, Rodríguez-Corsino M, Losada A. 2009. Heterochromatin protein 1 (HP1) proteins do not drive pericentromeric cohesin enrichment in human cells. PLoS One 4:e5118.

Shakya A, Park S, Rana N, King JT. 2020. Liquid-Liquid Phase Separation of Histone Proteins in Cells: Role in Chromatin Organization. Biophys $J$ 118:753-764.

Shin Y, Chang Y-C, Lee DSW, Berry J, Sanders DW, Ronceray P, Wingreen NS, Haataja M, Brangwynne CP. 2018. Liquid Nuclear Condensates Mechanically Sense and Restructure the Genome. Cell 175:1481-1491.e13.

Shivers JL, Feng J, van Oosten ASG, Levine H, Janmey PA, MacKintosh FC. 2020. Compression stiffening of fibrous networks with stiff inclusions. Proc Natl Acad Sci U S A 
117:21037-21044.

Shumaker DK, Dechat T, Kohlmaier A, Adam SA, Bozovsky MR, Erdos MR, Eriksson M, Goldman AE, Khuon S, Collins FS, Jenuwein T, Goldman RD. 2006. Mutant nuclear lamin A leads to progressive alterations of epigenetic control in premature aging. Proc Natl Acad Sci U S A 103:8703-8708.

Singh PB, Miller JR, Pearce J, Kothary R, Burton RD, Paro R, James TC, Gaunt SJ. 1991. A sequence motif found in a Drosophila heterochromatin protein is conserved in animals and plants. Nucleic Acids Res 19:789-794.

Stephens AD. 2020. Chromatin rigidity provides mechanical and genome protection. Mutat Res 821:111712.

Stephens AD, Banigan EJ, Adam SA, Goldman RD, Marko JF. 2017. Chromatin and lamin A determine two different mechanical response regimes of the cell nucleus. Mol Biol Cell 28:1984-1996.

Stephens AD, Banigan EJ, Marko JF. 2019a. Chromatin's physical properties shape the nucleus and its functions. Curr Opin Cell Biol 58:76-84.

Stephens AD, Haase J, Vicci L, Taylor RM 2nd, Bloom K. 2011. Cohesin, condensin, and the intramolecular centromere loop together generate the mitotic chromatin spring. J Cell Biol 193:1167-1180.

Stephens AD, Liu PZ, Banigan EJ, Almassalha LM, Backman V, Adam SA, Goldman RD, Marko JF. 2018. Chromatin histone modifications and rigidity affect nuclear morphology independent of lamins. Mol Biol Cell 29:220-233.

Stephens AD, Liu PZ, Kandula V, Chen H, Almassalha LM, Herman C, Backman V, O'Halloran T, Adam SA, Goldman RD, Banigan EJ, Marko JF. 2019b. Physicochemical mechanotransduction alters nuclear shape and mechanics via heterochromatin formation. Mol Biol Cell 30:2320-2330.

Strom AR, Emelyanov AV, Mir M, Fyodorov DV, Darzacq X, Karpen GH. 2017. Phase separation drives heterochromatin domain formation. Nature 547:241-245.

Style RW, Sai T, Fanelli N, ljavi M, Smith-Mannschott K, Xu Q, Wilen LA, Dufresne ER. 2018. Liquid-Liquid Phase Separation in an Elastic Network. Phys Rev X 8:011028.

Sun M, Biggs R, Hornick J, Marko JF. 2018. Condensin controls mitotic chromosome stiffness and stability without forming a structurally contiguous scaffold. Chromosome Res 26:277295.

Tamashunas AC, Tocco VJ, Matthews J, Zhang Q, Atanasova KR, Paschall L, Pathak S, Ratnayake R, Stephens AD, Luesch H, Licht JD, Lele TP. 2020. High-throughput gene screen reveals modulators of nuclear shape. Mol Biol Cell 31:1392-1402.

Tanaka F. 2002. Theoretical Study of Molecular Association and Thermoreversible Gelation in Polymers. Polym J 34:479-509.

Thiru A, Nietlispach D, Mott HR, Okuwaki M, Lyon D, Nielsen PR, Hirshberg M, Verreault A, Murzina NV, Laue ED. 2004. Structural basis of HP1/PXVXL motif peptide interactions and HP1 localisation to heterochromatin. EMBO J 23:489-499.

Uhler C, Shivashankar GV. 2018. Nuclear Mechanopathology and Cancer Diagnosis. Trends Cancer Res 4:320-331.

Vad-Nielsen J, Nielsen AL. 2015. Beyond the histone tale: HP1 1 deregulation in breast cancer epigenetics. Cancer Biol Ther 16:189-200.

Verschure PJ, van der Kraan I, de Leeuw W, van der Vlag J, Carpenter AE, Belmont AS, van Driel R. 2005. In vivo HP1 targeting causes large-scale chromatin condensation and enhanced histone lysine methylation. Mol Cell Biol 25:4552-4564.

Wang P, Dreger M, Madrazo E, Williams CJ, Samaniego R, Hodson NW, Monroy F, Baena E, Sánchez-Mateos P, Hurlstone A, Redondo-Muñoz J. 2018. WDR5 modulates cell motility and morphology and controls nuclear changes induced by a 3D environment. Proc Natl Acad Sci U S A 115:8581-8586. 
Williams JF, Surovtsev IV, Schreiner SM, Nguyen H. 2020. Phase separation enables heterochromatin domains to do mechanical work. bioRxiv.

Wreggett KA, Hill F, James PS, Hutchings A, Butcher GW, Singh PB. 1994. A mammalian homologue of Drosophila heterochromatin protein 1 (HP1) is a component of constitutive heterochromatin. Cytogenet Cell Genet 66:99-103.

Xie G, Walker RR 3rd, Irianto J. 2020. Nuclear mechanosensing: mechanism and consequences of a nuclear rupture. Mutat Res 821:111717.

Ye Q, Callebaut I, Pezhman A, Courvalin JC, Worman HJ. 1997. Domain-specific interactions of human HP1-type chromodomain proteins and inner nuclear membrane protein LBR. J Biol Chem 272:14983-14989.

Yi Q, Chen Q, Liang C, Yan H, Zhang Z, Xiang X, Zhang M, Qi F, Zhou L, Wang F. 2018. HP1 links centromeric heterochromatin to centromere cohesion in mammals. EMBO Rep 19. doi:10.15252/embr.201745484

Zhiteneva A, Bonfiglio JJ, Makarov A, Colby T, Vagnarelli P, Schirmer EC, Matic I, Earnshaw WC. 2017. Mitotic post-translational modifications of histones promote chromatin compaction. Open Biol 7. doi:10.1098/rsob.170076 